\title{
A SELECTION THEOREM FOR BANACH BUNDLES AND APPLICATIONS
}

\author{
ALDO J. LAZAR
}

\begin{abstract}
It is shown that certain lower semi-continuous maps from a paracompact space to the family of closed subsets of the bundle space of a Banach bundle admit continuous selections. This generalization of the the theorem of Douady, dal Soglio-Herault, and Hofmann on the fullness of Banach bundles has applications to establishing conditions under which the induced maps between the spaces of sections of Banach bundles are onto. Another application is to a generalization of the theorem of Bartle and Graves [3] for Banach bundle maps that are onto their images. Other applications of the selection theorem are to the study of the M-ideals of the space of bounded sections begun in [4] and continued in 9]. A class of Banach bundles that generalizes the class of trivial Banach bundles is introduced and some properties of these Banach bundles are discussed.
\end{abstract}

Date: April 17, 2016.

1991 Mathematics Subject Classification. Primary 46B20; Secondary 55R65, 58B05.

Key words and phrases. Banach bundles, Banach bundle maps, spaces of sections of Banach bundles, lower semi-continuous set-valued maps. 


\section{INTRODUCTION AND BASIC DEFINITIONS AND NOTATIONS}

The theory of Banach bundles, sometimes presented as continuous fields of Banach spaces, helped to advance the study of unitary representations of locally compact groups and of $C^{*}$-algebras. An early example of its usefulness and interest in these fields can be found in [7] and for later developments we mention [8]. Other beneficial connections of this theory are with the theory of Banach lattices. A short exposition on this aspect is given in the comprehensive monograph of Gierz [9] and more recent results appeared in [12]. The theory of Banach bundles provides an association of topology, the geometry of Banach spaces and operator theory, as exemplified in [9, Chapters 15 and 16] and [13].

We prove in this paper a generalization of Michael's well known selection theorem, [17, Theorem 3.2"], in the context of Banach bundles. This result, Theorem 2.9. also generalizes the theorems of Douady, L. dal Soglio-Herault, and K. H. Hofmann, see [8, Appendix C], on the existence of sufficiently many continuous crosssections in a Banach bundle. A particular case of Theorem 2.9] is [9, Proposition $15.13]$.

In Section 3 we consider Banach bundle maps (definition will follow) and the induced maps between the spaces of sections. We apply the selection theorem to establishing some conditions under which these later maps are onto their images. This investigation led to examining when a map between two Banach bundles is open. The section ends with the generalization of a theorem of Bartle and Graves on the existence of continuous right inverses for maps between Banach spaces. Here the context is of course maps between Banach bundles and we follow Michael [17] in using the selection theorem for deriving the existence of such an inverse.

Alfsen and Effros developed in [1] a structure theory for Banach spaces in which certain subspaces named M-ideals play a crucial role. The M-ideals of the Banach space of sections of a Banach bundle whose base space is compact Hausdorff were investigated in depth by Behrends [4] and Gierz [9]. In Section 4 we extend this investigation to the case when the base space is locally compact Hausdorff and the sections vanish at infinity.

A certain class of continuous Banach bundles is introduced in Section 5. the bundles in this class are called locally uniform. Every locally trivial Banach bundle 
is locally uniform but the converse is false. The locally uniform Banach bundles enjoy some nice properties. For instance we show in Proposition 5.7 that the quotient of a locally uniform Banach bundle by a locally uniform Banach subbundle is a continuous Banach bundle.

Some topological properties of the bundle space are treated in the Appendix. Conditions which insure that the bundle space is a Baire space are given. Also its paracompactness and its metrizability are discussed there.

Throughout of this paper the field of scalars is denoted by $\mathbb{K}$; it can be the field of the real numbers or the field of the complex numbers. A paracompact space is always considered to be Hausdorff. A topological space is locally paracompact if it has a cover with open subsets whose closures are paracompact. A map $\Phi$ from a topological space $T_{1}$ to the family of subsets of a topological space $T_{2}$ is called lower semi-continuous if for every open non-void subset $O$ of $T_{2}$ the set $\left\{t \in T_{1} \mid O \cap \Phi(t) \neq \emptyset\right\}$ is open in $T_{1}$.

For a Banach space $X, x \in X$, and $r$ a positive number we denote $B(x, r):=$ $\{y \in X \mid\|y-x\|<r\}$ and by $X_{r}$ the closed ball of $X$ whose center is at the origin with radius $r$. A closed subspace $Y$ of the Banach space $X$ is called an M-ideal of $X$ if its polar $Z_{1}$ in the dual of $X$ has a closed complement $Z_{2}$ such that $\left\|z_{1}+z_{2}\right\|=\left\|z_{1}\right\|+\left\|z_{2}\right\|$ for every $z_{i} \in Z_{i}, i=1,2$. A closed subspace $Y$ of $X$ is an M-ideal if and only if ithas the 3-ball property: if $\cap_{i=1}^{3} B\left(x_{i}, r_{i}\right) \neq \emptyset$ and $B\left(x_{i}, r_{i}\right) \cap Y \neq \emptyset, 1 \leq i \leq 3$, then $Y \cap \cap_{i=1}^{3} B\left(x_{i}, r_{i}\right) \neq \emptyset$.

By a Banach bundle $\xi:=(\mathcal{E}, p, T)$ we shall mean what in [5, pp. 8,9] is called an (H)Banach bundle. That is $x \rightarrow\|x\|$ is upper semi-continuous on the bundle space $\mathcal{E}$, see also [9, p.21]. We shall always suppose that the base space $T$ is Hausdorff. The fiber $p^{-1}(t)$ over $t \in T$ will be sometimes denoted $\mathcal{E}(t)$. The origin of the Banach space $\mathcal{E}(t)$ is denoted $0_{t}$ but if there can be no confusion just 0 will be used. The bundle $\xi$ is called a continuous Banach bundle if $x \rightarrow\|x\|$ is continuous on $\mathcal{E}$. The bundle space of a continuous Banach bundle is always Hausdorff, see 9. Proposition 16.4]. If $\mathcal{E}^{\prime}$ is a subset of $\mathcal{E}$ such that $\left.p\right|_{\mathcal{E}^{\prime}}$ is onto $T$ and open and each $p^{-1}(t) \cap \mathcal{E}^{\prime}$ is a closed subspace of $\mathcal{E}(t)$ then $\xi^{\prime}:=\left(\mathcal{E}^{\prime},\left.p\right|_{\mathcal{E}^{\prime}}, T\right)$ is a Banach bundle called a Banach subbundle of $\xi$. One can define an equivalence relation on $\mathcal{E}$ as follows: $x_{1}, x_{2}$ are equivalent if $p\left(x_{1}\right)=p\left(x_{2}\right)$ and $x_{1}-x_{2}$ belongs to 
$p^{-1}\left(p\left(x_{1}\right)\right)$. The quotient space, denoted $\mathcal{E} / \mathcal{E}^{\prime}$, is the union of all the quotient Banach spaces $\mathcal{E}(t) / \mathcal{E}^{\prime}(t), t \in T$. If we denote by $\tilde{p}$ the obvious map of $\mathcal{E} / \mathcal{E}^{\prime}$ onto $T$ then $\eta:=\left(\mathcal{E} / \mathcal{E}^{\prime}, \tilde{p}, T\right)$ is a Banach bundle by [9, Chapter 9]. The quotient map $q: \mathcal{E} \rightarrow \mathcal{E} / \mathcal{E}^{\prime}$ is open, see [9, 9.4].

Let $\xi:=(\mathcal{E}, p, T)$ be a Banach bundle. A continuous function $f: T \rightarrow \mathcal{E}$ is called a section of $\xi$ if $p(f(t))=t$ for every $t \in T$. The linear space of all the sections of $\xi$ is denoted $\Gamma(\xi)$; its subspace of all the bounded sections is denoted $\Gamma_{b}(\xi)$. This is a Banach space, the norm of $f \in \Gamma_{b}(\xi)$ being $\|f\|:=\sup _{t \in T}\|f(t)\|$. If $T$ is locally compact Hausdorff then the subspace $\Gamma_{0}(\xi)$ of $\Gamma_{b}(\xi)$ consisting of all the sections that vanish at infinity is of interest.

A full Banach bundle is a bundle such that for each $x \in \mathcal{E}$ there exists a section $f \in \Gamma(\xi)$ satisfying $f(p(x))=x$. In this definition one can replace $\Gamma(\xi)$ by $\Gamma_{b}(\xi)$ as observed in [5, p. 14] and even by $\Gamma_{0}(\xi)$ if $T$ is locally compact. For $f \in \Gamma(\xi), V$ an open subset of $T$, and $a>0$ the open subset

$$
U(f, V, a):=\{x \in \mathcal{E} \mid p(x) \in V,\|x-f(p(x))\|<a\}
$$

of $\mathcal{E}$ is called a tube. If $\xi$ is a full Banach bundle then the family of all the tubes forms a base for the topology of $\mathcal{E}$ by [5, p. 10].

A Banach bundle map from $\xi_{1}:=\left(\mathcal{E}_{1}, p_{1}, T\right)$ to $\xi_{2}:=\left(\mathcal{E}_{2}, p_{2}, T\right)$ is a continuous map $\varphi: \mathcal{E}_{1} \rightarrow \mathcal{E}_{2}$ such that $\varphi\left(p_{1}^{-1}(t)\right) \subset p_{2}^{-1}(t)$ for every $t \in T$ and the restriction of $\varphi$ to each fiber $p_{1}^{-1}(t)$ is linear. The map $\varphi$ is called an (isometric) isomorphism if $\varphi\left(\mathcal{E}_{1}\right)=\mathcal{E}_{2}$ and $\varphi$ is an isometry on every fiber.

\section{THE SELECTION THEOREM}

In this section we shall state and prove the selection theorem for set valued maps into a Banach bundle and we shall discuss some of its applications. The proof mimics closely the proofs given in [8, Appendix C] and [17]. A sequence of lemmas prepares the proof of the theorem itself.

From now on in this section $\xi:=(\mathcal{E}, p, T)$ is a fixed Banach bundle. Following [8. Appendix C] we shall say that a subset $U$ of $\mathcal{E}$ is $\varepsilon$-thin, $\varepsilon>0$, if $\left\|x-x^{\prime}\right\|<\varepsilon$ whenever $x, x^{\prime} \in \mathcal{E}$ and $p(x)=p\left(x^{\prime}\right)$.

Lemma 2.1. Let $t \in T, x_{1}, x_{2} \in p^{-1}(t)$ with $\left\|x_{1}-x_{2}\right\|<\varepsilon$. There is an open $\varepsilon$-thin set $U$ that contains the segment $\left[x_{1}, x_{2}\right]$. 
Proof. With $D:=\{(x, y) \in \mathcal{E} \times \mathcal{E} \mid p(x)=p(y)\}$, the set $\{(x, y) \mid\|x-y\|<\varepsilon\}$ is open since $(x, y) \rightarrow\|x-y\|$ is upper semi-continuous. There is an open subset $O$ of $\mathcal{E} \times \mathcal{E}$ such that

$$
\left[x_{1}, x_{2}\right] \times\left[x_{1}, x_{2}\right] \subset\{(x, y) \mid\|x-y\|\}=O \cap D .
$$

By [18, p. 171] there is an open set $U \subset \mathcal{E}$ such that

$$
\left[x_{1}, x_{2}\right] \times\left[x_{1}, x_{2}\right] \subset U \times U \subset O .
$$

Obviously $U$ is $\varepsilon$-thin.

We enlarge now our setting with a completely regular topological space $S$ and a continuous open map $\pi$ of $S$ onto $T$. A function $f: S \rightarrow \mathcal{E}$ is called admissible if $p \circ f=\pi$. Let $\varepsilon>0$. A function $f: S \rightarrow \mathcal{E}$ is called $\varepsilon$-continuous at $s \in S$ if there are a neighbourhood $V$ of $s$ and an $\varepsilon$-thin neighbourhood $U$ of $f(s)$ such that $f(V) \subset U$. If $f$ is $\varepsilon$-continuous at all the points of $S$ then it is called shortly $\varepsilon$-continuous.

Lemma 2.2. If an admissible function $f$ is $\varepsilon$-continuous for all $\varepsilon>0$ then it is continuous.

Proof. Let $s \in S$ and and suppose that $V_{n}$ is a neighbourhood of $s$ and $U_{n}$ is an $1 / n$-thin neighbourhood of $f(s)$, with $f\left(V_{n}\right) \subset U_{n}, n \in \mathbb{N}$. We have $f\left(V_{n} \cap\right.$ $\left.V_{n+1}\right) \subset U_{n} \cap U_{n+1}$ so there is no loss of generality if we suppose that $\left\{U_{n}\right\}$ is decreasing. Let now $W$ be a neighbourhood of $f(s)$. By [5, p. 10] there are a natural number $n$ and a neighbourhood $V$ of $\pi(s)$ such that $U_{n} \cap p^{-1}(V) \subset W$. The neighbourhood $V \cap \pi\left(V_{n}\right)$ of $p(f(s))=\pi(s)$ satisfies $U_{n} \cap p^{-1}\left(V \cap \pi\left(V_{n}\right)\right) \subset W$. The neighbourhood $V_{n} \cap \pi^{-1}(V)$ os $s$ satisfies $f\left(V_{n} \cap \pi^{-1}(V)\right) \subset W$. Indeed, if $s^{\prime} \in V_{n} \cap \pi^{-1}(V) \subset V_{n}$ then $f\left(s^{\prime}\right) \in U_{n}$. Moreover, $p\left(f\left(s^{-1}\right)\right)=\pi\left(s^{\prime}\right) \in \pi\left(V_{n}\right) \cap V$; hence $f\left(s^{\prime}\right) \in p^{-1}\left(\pi\left(V_{n}\right) \cap V\right) \subset p^{-1}(V)$. Thus $f\left(s^{\prime}\right) \in U_{n} \cap p^{-1}(V) \subset W$ and the continuity of $f$ at $s$ is established.

Lemma 2.3. Let $G$ be an open subset of $S$ and $\left\{U_{i}\right\}_{i=1}^{n}$ be open subsets of $\mathcal{E}$ such that $p\left(U_{i}\right)=\pi(G), 1 \leq i \leq n$. Suppose that $\left\{\varphi_{i}\right\}_{i=1}^{n}$ are continuous scalar functions 
on $G$ with $\varphi_{1}(s) \neq 0$ for each $s \in G$. Then the set

$$
W:=\left\{\sum \varphi_{i}(s) x_{i} \mid s \in G, x_{i} \in U_{i}, p\left(x_{i}\right)=\pi(s), 1 \leq i \leq n\right\}
$$

is open in $\mathcal{E}$.

Proof. Consider the set

$$
Z:=\left\{\left(s, x_{1} \ldots, x_{n}\right) \mid s \in G, x_{i} \in \mathcal{E}, p\left(x_{i}\right)=\pi(s), 1 \leq i \leq n\right\}
$$

endowed with the relative topology inherited from $G \times p^{-1}(\pi(G))^{n}$. A basis for this topology consists of all the sets of the form $O:=\left(V \times V_{1} \times \ldots V_{n}\right) \cap Z$ where $V \subset G, V_{i} \subset p^{-1}(\pi(G)), 1 \leq i \leq n$, are open. Thus

$$
O:=\left\{\left(s, x_{1}, \ldots x_{n}\right) \mid s \in V, x_{i} \in V_{i}, p\left(x_{i}\right)=\pi(s), 1 \leq i \leq n\right\} .
$$

The map $\alpha$ of $Z$ onto itself given by $\alpha\left(s, x_{1}, \ldots x_{n}\right):=\left(s, \sum \varphi_{i}(s) x_{i}, x_{2}, \ldots x_{n}\right)$ is a homeomorphism. Indeed it is continuous and its inverse maps $\left(s, y_{1}, \ldots y_{n}\right)$ to $\left(s, 1 / \varphi_{1}(s)\left(y_{1}-\sum_{i=2}^{n} \varphi_{i}(s) y_{i}\right), y_{2}, \ldots y_{n}\right)$. The map $\beta$ of $Z$ into $\mathcal{E}$ given by $\beta\left(s, x_{1}, \ldots x_{n}\right):=x_{1}$ is open. Indeed, if $O$ is as in (1) then

$$
\beta(O)=V_{1} \cap p^{-1}\left(\pi(V) \cap \cap_{i=1}^{n} p\left(V_{i}\right)\right)
$$

that is open. Now $W=\beta\left(\alpha\left(\left(G \times U_{1}, \ldots U_{n}\right) \cap Z\right)\right)$ is obviously open.

Lemma 2.4. Suppose $\varphi_{i}, 1 \leq i \leq n$, are continuous functions from $S$ to $[0,1]$ with $\sum \varphi_{i}=1$. Let $\varepsilon>0$. If $f_{i}, 1 \leq i \leq n$ are admissible and $\varepsilon$-continuous at $s_{0} \in S$ then $f:=\sum \varphi_{i} f_{i}$ is admissible and $\varepsilon$-continuous at $s_{0}$.

Proof. We have to prove only that $f$ is $\varepsilon$-continuous at $s_{0}$. There is no loss of generality if we suppose $\varphi_{1}\left(s_{0}\right) \neq 0$. There exist $\varepsilon$-thin open neighbourhoods $U_{i}$ of $f_{i}\left(s_{0}\right)$ and open neighbourhoods $V_{i}$ of $s_{0}$ such that $f_{i}\left(V_{i}\right) \subset U_{i}, 1 \leq i \leq n$. By taking $\cap_{i=1}^{n} V_{i}$ and further reducing it if needed one gets an open neighbourhood $V$ of $s_{0}$ such that $f_{i}(V) \subset U_{i}, 1 \leq i \leq n$, and $\varphi(s)>0$ if $s \in V$. From $f_{i}(V) \subset U_{i}$ and the admissibility of $f_{i}$ we get $f_{i}(V) \subset p^{-1}(\pi(V)) \cap U_{i}$ and $p\left(p^{-1}(\pi(V)) \cap U_{i}\right)=\pi(V)$, $1 \leq i \leq n$. The set $p^{-1}(\pi(V)) \cap U_{i}$ is an open neighbourhood of $f_{i}\left(s_{0}\right)$. The set

$$
W:=\left\{\sum \varphi_{i}(s) x_{i} \mid s \in V, x_{i} \in p^{-1}(\pi(V)) \cap U_{i}, p\left(x_{i}\right)=\pi(s), 1 \leq i \leq n\right\}
$$

is open in $\mathcal{E}$ by Lemma 2.3 and $f\left(s_{0}\right) \in W$. By the above $f(V) \subset W$ and it remains to show that $W$ is $\varepsilon$-thin. To this end, let $x^{\prime}, x^{\prime \prime} \in W$ with $p\left(x^{\prime}\right)=p\left(x^{\prime \prime}\right)=\pi(s)$ for 
some $s \in V$. Then $x^{\prime}=\sum \varphi_{i}(s) x_{i}^{\prime}$ with $x_{i}^{\prime} \in U_{i}, p\left(x_{i}^{\prime}\right)=\pi(s)$ and $x^{\prime \prime}=\sum \varphi_{i}(s) x_{i}^{\prime \prime}$ with $x_{i}^{\prime \prime} \in U_{i}, p\left(x_{i}^{\prime \prime}\right)=\pi(s), 1 \leq i \leq n$. Each $U_{i}$ is $\varepsilon$-thin so $\left\|x^{\prime}-x^{\prime \prime}\right\|<\varepsilon$ and we conclude that $W$ is $\varepsilon$-thin.

Lemma 2.5. Let $x_{0} \in \mathcal{E}, s_{0} \in S$ with $\pi\left(s_{0}\right)=p\left(x_{0}\right)$. There exists an $\varepsilon$-continuous admissible function $f: S \rightarrow \mathcal{E}$ such that $f\left(s_{0}\right)=x_{0}$.

Proof. Let $U$ be an $\varepsilon$-thin open neighbourhood of $x_{0}$ and set $V:=\pi^{-1}(p(U))$ which is an open neighbourhood of $s_{0}$. Define $g: V \rightarrow \mathcal{E}$ so that $g\left(s_{0}\right)=x_{0}$ and $g(s) \in U$ with $p(g(s))=\pi(s)$ for $s \in V$. Let $W$ be an open neighbourhood of $s_{0}$ such that $\bar{W} \subset V$ and $\varphi: S \rightarrow[0,1]$ a continuous function such that $\varphi\left(s_{0}\right)=1$ and $\left.\varphi\right|_{S \backslash \bar{W}} \equiv 0$. Set

$$
f(s):= \begin{cases}\varphi(s) g(s), & \text { if } s \in V, \\ 0, & \text { if } s \notin V .\end{cases}
$$

Then on $V, f=\varphi g+(1-\varphi) 0$. Thus $f$ is $\varepsilon$-continuous on $V$ by Lemma 2.4 and trivially $\varepsilon$-continuous on $S \backslash \bar{W}$. Clearly $f$ is an admissible function.

From here on, until the end of the proof of Theorem 2.9, $S$ is a paracompact space and $\Phi$ is a lower semi-continuous map from $S$ to the family of non-void closed subsets of $\mathcal{E}$ such that $\Phi(s)$ is a convex subset of $p^{-1}(\pi(s))$ for every $s \in S$.

Lemma 2.6. Given $s_{0} \in S$ and $\varepsilon>0$ there are an $\varepsilon$-continuous admissible function $f$ and a neighbourhood $V$ of $s_{0}$ such that $B(f(s), \varepsilon) \cap \Phi(s) \neq \emptyset$ for every $s \in S$.

Proof. Let $x_{0} \in \Phi\left(s_{0}\right)$ and $f$ be an $\varepsilon$-continuous admissible function such that $f\left(s_{0}\right)=x_{0}$ as given by Lemma 2.5. There are an $\varepsilon$-thin neighbourhood $U$ of $x_{0}$ and a neighbourhood $V_{1}$ of $s_{0}$ such that $f\left(V_{1}\right) \subset U$. The set $V_{2}:=\{s \in S \mid U \cap \Phi(s) \neq \emptyset\}$ is an open neighbourhood of $s_{0}$. Set $V:=V_{1} \cap V_{2}$. Then, for each $s \in V$ there is $x \in U \cap \Phi(s)$ and $\|f(s)-x\|<\varepsilon$ since $f(s) \in U$ and $U$ is an $\varepsilon$-thin set.

Now we have reached the main approximation lemma. Its statement consists of two assertions with similar proofs.

Lemma 2.7. Let $\varepsilon>0$. 
(i) There is an E-continuous admissible function $g$ such that $B(g(s), \varepsilon) \cap \Phi(s) \neq \emptyset$ for every $s \in S$.

(ii) Given an $\varepsilon$-continuous admissible function $f$ such that $B(f(s), \varepsilon) \cap \Phi(s) \neq \emptyset$ for every $s \in S$ there is an $\varepsilon / 2$-continuous admissible function $g$ such that $\|f(s)-g(s)\|<2 \varepsilon$ and $B(g(s), \varepsilon / 2) \cap \Phi(s) \neq \emptyset$ for every $s \in S$.

Proof. We begin with the proof of (ii). Given $f$ as above, the first step of the proof is to show that for every $s \in S$ there are an open neighbourhood $V$ and an $\varepsilon / 2$-continuous admissible function $h$ such that $B\left(h\left(s^{\prime}\right), \varepsilon / 2\right) \cap \Phi\left(s^{\prime}\right) \neq \emptyset$ and $\left\|f\left(s^{\prime}\right)-h\left(s^{\prime}\right)\right\|<2 \varepsilon$ for each $s^{\prime} \in V$. Let $s \in S$ and choose $x \in B(f(s), \varepsilon) \cap \Phi(s)$. There is an $\varepsilon$-thin open neighbourhood $U_{1}$ of $f(s)$ and an open neighbourhood $V_{1}$ of $s$ such that $f\left(V_{1}\right) \subset U_{1}$. Let $y=3 / 4 x+1 / 4 f(s)$. Lemma 2.5 yields an $\varepsilon / 4$ continuous admissible function $h$ such that $h(s)=y$. We are going to show that $h$ satisfies our claim.

There are an $\varepsilon / 4$-thin open neighbourhood $U_{2}$ of $y$ and an open neighbourhood $V_{2}$ of $s$ such that $h\left(V_{2}\right) \subset U_{2}$. By using Lemma 2.1 we get an $\varepsilon / 4$-thin open set $U_{3}$ that contains the segment $[y, x]$ and an open $3 \varepsilon / 4$-thin set $U_{4}$ that contains the segment $[y, f(s)]$. Denote $V^{\prime}:=\left\{s^{\prime} \mid U_{3} \cap \Phi\left(s^{\prime}\right) \neq \emptyset\right\}$, an open neighbourhood of $s$ since $x \in U_{3} \cap \Phi(s)$. Set now

$$
V:=\pi^{-1}\left(p\left(U_{2} \cap U_{3} \cap U_{4}\right)\right) \cap \pi^{-1}\left(p\left(U_{1} \cap U_{4}\right)\right) \cap V_{1} \cap V_{2} \cap V^{\prime} .
$$

We have $s \in \pi^{-1}\left(p\left(U_{2} \cap U_{3} \cap U_{4}\right)\right)$ since $y \in U_{2} \cap U_{3} \cap U_{4}$ and $p(y)=p(f(s))=\pi(s)$. Moreover, $f(s) \in U_{1} \cap U_{4}$ hence $s \in \pi^{-1}\left(p\left(U_{1} \cap U_{4}\right)\right)$. We conclude that $V$ is an open neighbourhood of $s$. If $s^{\prime} \in V \subset V_{1}$ then $f\left(s^{\prime}\right) \in U_{1}$ and $\pi\left(s^{\prime}\right) \in p\left(U_{1} \cap U_{4}\right)$. Thus there is $x_{1} \in U_{1} \cap U_{4}$ with $p\left(x_{1}\right)=\pi\left(s^{\prime}\right)$ and

$$
\left\|f\left(s^{\prime}\right)-x_{1}\right\|<\varepsilon
$$

since $U_{1}$ is $\varepsilon$-thin. For $s^{\prime} \in V$ we have $\pi\left(s^{\prime}\right) \in p\left(U_{2} \cap U_{3} \cap U_{4}\right)$ hence there is $x_{2} \in U_{2} \cap U_{3} \cap U_{4}$ such that $p\left(x_{2}\right)=\pi\left(s^{\prime}\right)$. From $s^{\prime} \in V \subset V_{2}$ we get $h\left(s^{\prime}\right) \in U_{2}$ and

$$
\left\|h\left(s^{\prime}\right)-x_{2}\right\|<\varepsilon / 4 .
$$

The set $U_{4}$ is $3 / 4 \varepsilon$-thin so

$$
\left\|x_{1}-x_{2}\right\|<3 / 4 \varepsilon .
$$


Finally, if $s^{\prime} \in V \subset V^{\prime}$ then there is $x_{3} \in U_{3} \cap \Phi\left(s^{\prime}\right)$ and we have

$$
\left\|x_{2}-x_{3}\right\|<\varepsilon / 4
$$

since $U_{3}$ is $\varepsilon / 4$-thin. From (44) and (6) we get

$$
\left\|h\left(s^{\prime}\right)-x_{3}\right\| \leq\left\|h\left(s^{\prime}\right)-x_{2}\right\|+\left\|x_{2}-x_{3}\right\|<\varepsilon / 2
$$

Therefore, if $s^{\prime} \in V$ then $B\left(h\left(s^{\prime}\right), \varepsilon / 2\right) \cap \Phi\left(s^{\prime}\right) \neq \emptyset$. Also, if $s^{\prime} \in V$ we get from (4), (15) and (3) that

$$
\left\|h\left(s^{\prime}\right)-f\left(s^{\prime}\right)\right\| \leq\left\|h\left(s^{\prime}\right)-x_{2}\right\|+\left\|x_{2}-x_{1}\right\|+\left\|x_{1}-f\left(s^{\prime}\right)\right\|<\varepsilon / 4+3 \varepsilon / 4+\varepsilon=2 \varepsilon
$$

and the claim is established.

As mentioned above, what has been done up to this point is irrelevant for (i). To obtain the proof of (i) from what follows one has to ignore every affirmation about the function $f$.

We know now that there exist an open covering $\left\{V_{\alpha}\right\}_{\alpha \in \mathcal{A}}$ of $S$ and admissible $\varepsilon / 2$-continuous functions $h_{\alpha}: S \rightarrow \mathcal{E}$ such that $B\left(h_{\alpha}(s), \varepsilon / 2\right) \cap \Phi(s) \neq \emptyset$ and $\left\|f(s)-h_{\alpha}\right\|<2 \varepsilon$ for every $s \in V_{\alpha}$. For proving (i) one obtains this from Lemma 2.6. We may and shall suppose that the covering of $S$ is locally finite. Let $\left\{\varphi_{\alpha}\right\}$ be a partition of unity subordinated to $\left\{V_{\alpha}\right\}$. and define $g:=\sum_{\alpha} \varphi_{\alpha} h_{\alpha}$. Each point $s \in S$ has a neighbourhood $O_{s}$ such that there exists a finite set $\mathcal{F}_{s} \subset \mathcal{A}$ with the property that $O_{s} \cap V_{\alpha}=\emptyset$ is $\alpha \notin \mathcal{F}_{s}$. Thus $g$ is well defined, admissible and $\varepsilon / 2$-continuous by Lemma 2.4. We have $B(g(s), \varepsilon / 2) \cap \Phi(s) \neq \emptyset$ for every $s \in S$. Indeed, choose $z_{\alpha} \in B\left(h_{\alpha}(s), \varepsilon / 2\right) \cap \Phi(s)$ for $\alpha \in \mathcal{F}_{s}$. Then $z:=\sum_{\alpha \in \mathcal{F}_{s}} z_{\alpha} \in \Phi(s)$ and

$$
\|g(s)-z\| \leq \sum_{\alpha \in \mathcal{F}_{s}} \varphi_{\alpha}(s)\left\|h_{\alpha}(s)-z_{\alpha}\right\|<\varepsilon / 2
$$

Furthermore,

$\|g(s)-f(s)\| \leq\left\|\sum_{\alpha \in \mathcal{F}_{s}} \varphi_{\alpha}(s) h_{\alpha}(s)-\sum_{\alpha \in \mathcal{F}_{s}} \varphi_{\alpha}(s) f(s)\right\| \leq \sum_{\alpha \in \mathcal{F}_{s}} \varphi_{\alpha}(s)\left\|h_{\alpha}(s)-f(s)\right\|<2 \varepsilon$ and the proof is complete.

Lemma 2.8. Let $\left\{f_{n}\right\}$ be a sequence of admissible $\varepsilon$-continuous $(\varepsilon>0)$ functions, uniformly convergent on $S$. Then its limit $f$ is admissible and $2 \varepsilon$-continuous. 
Proof. Clearly $f$ is admissible; we have only to prove that it is $2 \varepsilon$-continuous. Let $n$ be such that $\left\|f(s)-f_{n}(s)\right\|<\varepsilon / 2$ for every $s \in S$. For $s_{0} \in S$ there are a neighbouhood $V$ of $s_{0}$ and a neighbourhood $U^{\prime}$ of $f_{n}\left(s_{0}\right)$ such that $f_{n}(V) \subset U^{\prime}$. Now, $f_{n}$ is admissible so $\pi(V) \subset U^{\prime}$. Put $U:=U^{\prime} \cap p^{-1}(\pi(V))$ and

$$
W:=\{x+y \mid p(x)=p(y) \in \pi(V),\|x\|<\varepsilon / 2, y \in U\} .
$$

Then Lemma 2.3 yields that $W$ is an open neighbourhoo of $f\left(s_{0}\right)$ and it is easily seen that it is $2 \varepsilon$-thin. If $s \in V$ then $f(s)=f_{n}(s)+\left(f(s)-f_{n}(s)\right) \in W$. We conclude that $f$ is $2 \varepsilon$-continuous at $s_{0}$.

In all the applications but one of the theorem that follows the setup will be somewhat simpler; we shall have $S=T$ and $\pi$ will be the identity map of $T$.

Theorem 2.9. Let $\xi:=(p, \mathcal{E}, T)$ be a Banach bundle, $S$ a paracompact topological space, $\pi$ a continuous open map of $S$ onto $T$ and $\Phi$ a lower semi-continuous map from $S$ to the family of non-empty closed subsets of $\mathcal{E}$ such that $\Phi(s)$ is a convex subset of the fiber $p^{-1}(\pi(s))$ for every $s \in S$. Then there exists a continuous admissible function $f: S \rightarrow \mathcal{E}$ such that $f(s) \in \Phi(s)$ for every $s \in S$. Moreover, if $s_{0} \in S$ and $x_{0} \in \Phi\left(s_{0}\right)$ then $f$ can be chosen so that $f\left(s_{0}\right)=x_{0}$.

Proof. By Lemma 2.7 (i) there exists an $1 / 2$-continuous admissible function $f_{1}$ such that $B\left(f_{1}(s), 1 / 2\right) \cap \Phi(s) \neq \emptyset$ for every $s \in S$. We proceed now by induction. Suppose that admissible functions $\left\{f_{i}\right\}_{i=1}^{n}$ are given such that each $f_{i}$ is $1 / 2^{i}$ continuous, satisfies $B\left(f_{i}(s), 1 / 2^{i}\right) \cap \Phi(s) \neq \emptyset$ and $\left\|f_{i}(s)-f_{i+1}(s)\right\|<1 / 2^{i-1}$ for every $s \in S$ and $1 \leq i \leq n-1$. By Lemma 2.7 (ii) there exists an $i / 2^{n+1}$ continuous admissible function $f_{n+1}$ such that $B\left(f_{n+1}(s), 1 / 2^{n+1}\right) \cap \Phi(s) \neq \emptyset$ and $\left\|f_{n}(s)-f_{n+1}(s)\right\|<1 / 2^{n-1}$ for every $s \in S$. The sequence $\left\{f_{n}\right\}$ is uniformly convergent on $S$. The limit $f$ is admissible and $1 / 2^{n}$-continuous for $n \geq 1$ by Lemma 2.8. Thus $f$ is continuous on $S$ by Lemma 2.2. Obviously $f(s) \in \Phi(s)$ for each $s \in S$.

The second assertion of the theorem follows from the first by considering the lower semi-continuous set valued map

$$
\left.\Phi^{(} s\right):=\left\{\begin{array}{l}
\Phi(s), s \neq s_{0}, \\
x_{0}, s=s_{0}
\end{array}\right.
$$


Theorem [2.9] is a generalization of [9, Proposition 15.13] where the case $S=$ $T$, a compact Hausdorff space, $\pi$ being the identity map of $T$, is discussed. By considering a trivial Banach bundle one can deduce from Theorem 2.9 the existence of the selection part of Michael's [17, Theorem 3.2"]. Another known result that can be deduced from Theorem 2.9 is the observation on p. 15 of [5] that partial section over closed subsets of the base space can be extended to sections over the entire base space. We state below the precise result.

Corollary 2.10. Let $\xi:=(p, \mathcal{E}, T)$ be a Banach bundle with paracompact base space, $A$ a closed subset of $T$ and $\varphi: A \rightarrow \mathcal{E}$ a section of $\xi \mid A$. Then there exists a section $\tilde{\varphi}: T \rightarrow \mathcal{E}$ of $\xi$ that extends $\varphi$. Moreover, if $\varphi$ is bounded then there exists a bounded extension $\tilde{\varphi}$. If $\xi:=(\mathcal{E}, p, T)$ is a Banach bundle with locally compact Hausdorff base space, $A$ a compact subset of $T$ and $\varphi$ a section of $\xi \mid A$ then there is a section $\tilde{\varphi} \in \Gamma_{0}(\xi)$ that extends $\varphi$.

Proof. The first claim is obtained by applying Theorem 2.9 to the set valued map

$$
\Phi(t):=\left\{\begin{array}{l}
\{\varphi(t)\}, t \in A \\
p^{-1}(t), t \in T \backslash A .
\end{array}\right.
$$

To get a bounded extension in case $\varphi$ is bounded one multiplies a section that extends $\varphi$ by a bounded continuous scalar valued function that is constantly equal to 1 on $A$. Finally, when $T$ is locally compact Hausdorff and $A$ is compact one extends $\varphi$ to a section $\varphi^{\prime}$ of $\xi \mid \bar{V}, V$ being an open neighbourhood of $A$ with compact closure. With $f: T \rightarrow[0,1]$ a continuous function such that $f \mid A \equiv 1$ and $f \mid T \backslash V \equiv 0$ one defines

$$
\tilde{\varphi}(t):=\left\{\begin{array}{l}
f(t) \varphi^{\prime}(t), t \in V \\
0_{t}, t \in T \backslash V .
\end{array}\right.
$$

Of course, one can deduce from Corollary 2.10 the well known theorems of A. Douady, L. dal Soglio-Herault and K. H. Hofmann ([8, pp. 640-641]) on the fullness of Banach bundles with paracompact or locally compact Hausdorff base spaces. 


\section{BANACH BUNDLE MAPS}

We shall now discuss Banach bundle maps and the way they affect various spaces of sections. We shall end this chapter with an extension of a well known result of Bartle and Graves [3] to Banach bundle maps.

Let $\xi_{1}:=\left(\mathcal{E}_{1}, p_{1}, T\right)$ and $\xi_{2}:=\left(\mathcal{E}_{2}, p_{2}, T\right)$ be Banach bundles. Recall that a Banach bundle map from $\xi_{1}$ to $\xi_{2}$ is a continuous map $\varphi: \mathcal{E}_{1} \rightarrow \mathcal{E}_{2}$ such that $p_{2} \circ \varphi=p_{1}$ and which acts linearly on each fiber of $\xi_{1}$. The restriction of $\varphi$ to $p_{1}^{-1}(t), t \in T$, is a bounded linear operator into $p_{2}^{-1}(t)$ which we shall denote by $\varphi_{t}$. The Banach bundle map $\varphi$ induces a linear map $\tilde{\varphi}: \Gamma\left(\xi_{1}\right) \rightarrow \Gamma\left(\xi_{2}\right)$ as follows:

$$
\tilde{\varphi}(f)(t):=\varphi(f(t)), f \in \Gamma\left(\xi_{1}\right), t \in T .
$$

This map $\tilde{\varphi}$ need not be onto $\Gamma\left(\xi_{2}\right)$ even if $\varphi$ is onto $\mathcal{E}_{2}$. To illustrate this we shall present an example inspired by one in 2]. In order to have $\tilde{\varphi}\left(\Gamma\left(\xi_{1}\right)=\Gamma\left(\xi_{2}\right)\right.$ one has to require $\varphi$ to be open.

Example 3.1. Let

$$
\left.\left.\mathcal{E}_{1}:=[0,1] \times(\mathbb{K} \times \mathbb{K}), \mathcal{E}_{2}:=(\{0\} \times(\{0\} \times \mathbb{K})) \cup((0,1] \times(\mathbb{K} \times \mathbb{K})) \mathbb{K}\right)\right)
$$

and $p$ be the projection of $\mathcal{E}_{1}$ onto $[0,1]$. We give the linear space $\{t\} \times(\mathbb{K} \times \mathbb{K}) \subset \mathcal{E}_{1}$ the norm $\|(t,(x, y))\|:=\max (|x|,|y|)$. Then $\xi_{1}:=\left(\mathcal{E}_{1}, p,[0,1]\right)$ is a trivial Banach bundle and $\xi_{2}:=\left(\mathcal{E}_{2}, p \mid \mathcal{E}_{2},[0,1]\right)$ a Banach subbundle of $\xi_{1}$. Define the Banach bundle map $\varphi: \mathcal{E}_{1} \rightarrow \mathcal{E}_{2}$ by $\varphi(t,(x, y)):=(t,(t x, y))$. Then $\varphi\left(\mathcal{E}_{1}\right)=\mathcal{E}_{2}$. It is obvious that the section $f(t):=\left(t,\left(t^{1 / 2}, 1\right)\right)$ of $\Gamma\left(\xi_{2}\right)$ has no preimage in $\Gamma\left(\xi_{1}\right)$.

Theorem 3.2. Let $\xi_{i}:=\left(\mathcal{E}_{i}, p_{1}, T\right), i=1,2$, be Banach bundles with $T$ paracompact and $\varphi:=\mathcal{E}_{1} \rightarrow \mathcal{E}_{2}$ an open Banach bundle map onto $\mathcal{E}_{2}$. Then $\tilde{\varphi}\left(\Gamma\left(\xi_{1}\right)\right)=\Gamma\left(\xi_{2}\right)$.

Proof. Let $f \in \Gamma\left(\xi_{2}\right)$ and define $\Phi(t):=\varphi^{-1}(f(t)), t \in T$. We are going to show that $\Phi$ is lower semi-continuous. To this end let $U \subset \mathcal{E}_{1}$ be open and suppose $U \cap \Phi\left(t_{0}\right) \neq \emptyset$. Thus there exists $x_{0} \in U$ with $p\left(x_{0}\right)=t_{0}$ and $\varphi\left(x_{0}\right)=f\left(t_{0}\right)$. The set $\varphi(U)$ is open in $\mathcal{E}_{2}$ therefore there exists an open neighbourhood $V$ of $t_{0}$ such that $f(V) \subset \varphi(U)$. It follows that $t_{0} \in V \subset\{t \in T \mid U \cap \Phi(t) \neq \emptyset\}$ and we conclude that the latter set is open. By Theorem $2.9 \Phi$ admits a continuous selection $g$. This is a section of $\xi_{1}$ that satisfies $\tilde{\varphi}(g)=f$. 
Thus it seems to be of some interest to establish conditions for a Banach bundle map which is onto the bundle space of the image to be open.

Proposition 3.3. Let $\xi_{1}:=\left(\mathcal{E}_{1}, p_{1}, T\right)$ be a full Banach bundle and $\varphi$ a Banach bundle map of $\xi_{1}$ onto $\xi_{2}:=\left(\mathcal{E}_{2}, p_{2}, T\right)$. Then $\varphi$ is open if and only if there exists an open cover $\left\{V_{\alpha}\right\}_{\alpha \in \mathcal{A}}$ of $T$ and positive numbers $\left\{m_{\alpha}\right\}_{\alpha \in \mathcal{A}}$ such that for every $y \in p_{2}^{-1}\left(V_{\alpha}\right)$ there is $x \in \mathcal{E}_{1}$ that satisfies $\varphi(x)=y$ and $\|x\| \leq m_{\alpha}\|y\|, \alpha \in \mathcal{A}$,

Proof. Suppose that $\varphi$ satisfies the condition; we are going to show that $\varphi$ is open on $p_{1}^{-1}\left(V_{\alpha}\right), \alpha \in \mathcal{A}$, and this will prove the 'if' part of the statement. So let $U \subset p_{1}^{-1}\left(V_{\alpha}\right)$ be an open set and $y_{0} \in \varphi(U)$. Denote $t_{0}:=p_{2}\left(y_{0}\right) \in V_{\alpha}$ and let $x_{0} \in U$ be such that $\varphi\left(x_{0}\right)=y_{0}$ and $f \in \Gamma\left(\xi_{1}\right)$ with $f\left(t_{0}\right)=x_{0}$. There exist an open neighbourhood $V$ of $t_{0}$ in $V_{\alpha}$ and $\varepsilon>0$ such that

$$
x_{0} \in\left\{x \in p_{1}^{-1}\left(V_{\alpha}\right) \mid p_{1}(x) \in V,\left\|x-f\left(p_{1}(x)\right)\right\|<\varepsilon\right\} \subset U .
$$

If not then there would exist a net $\left\{x_{\iota}\right\}$ in $p_{1}^{-1}\left(V_{\alpha}\right) \backslash U$ such that $\left\{p_{1}\left(x_{\iota}\right)\right\}$ converges to $t_{0}$ and $\left\|x_{\iota}-f\left(p_{1}\left(x_{\iota}\right)\right)\right\| \rightarrow 0$. But that means $x_{\iota} \rightarrow f\left(t_{0}\right)=x_{0} \in U$, a contradiction. Hence $V$ and $\varepsilon>0$ as claimed indeed exist. Put $\tilde{f}:=\tilde{\varphi}(f)$. We claim now that the open neighbourhood

$$
U^{\prime} ;=\left\{y \in p_{2}^{-1}\left(V_{\alpha}\right) \mid p_{2}(y) \in V,\left\|y-\tilde{f}\left(p_{2}(y)\right)\right\|<\varepsilon / m_{\alpha}\right\}
$$

of $y_{0}$ is contained in $\varphi(U)$. Indeed, let $y \in U^{\prime}$. By our assumption, there exists $x \in p_{1}^{-1}\left(p_{2}(y)\right)$ such that $\varphi(x)=y-\tilde{f}\left(p_{2}(y)\right)$ and

$$
\|x\| \leq m_{\alpha}\left\|y-\tilde{f}\left(p_{2}(y)\right)\right\|<\varepsilon .
$$

Since

$$
\left\|\left(x+f\left(p_{2}(y)\right)\right)-f\left(p_{2}(y)\right)\right\|=\|x\|<\varepsilon
$$

we get $x+f\left(p_{2}(y)\right) \in U$ and $y=\varphi\left(x+f\left(p_{2}(y)\right)\right) \in \varphi(U)$ as needed.

Suppose now that $\varphi$ is open. Let $t \in T$. The set $\varphi\left(\left\{x \in \mathcal{E}_{1} \mid\|x\|<1\right\}\right)$ is an open neighbourhood of $0_{t}$. There exist an open neighbourhood $V_{t}$ of $t$ and a positive number $k_{t}$ such that

$$
\left\{y \in \mathcal{E}_{2} \mid p_{2}(y) \in V_{t},\|y\|<k_{t}\right\} \subset \varphi\left(\left\{x \in \mathcal{E}_{1} \mid\|x\|<1\right\}\right)
$$

If not then there would exist a net $\left\{y_{\iota}\right\}$ in $\mathcal{E}_{2} \backslash \varphi\left(\left\{x \in \mathcal{E}_{1} \mid\|x\|\right\}\right)$ such that $\left\{p_{2}\left(y_{\iota}\right)\right\}$ converges to $t$ and $\left\|y_{\iota}\right\| \rightarrow 0$. But then $y_{\iota} \rightarrow 0_{t}$, a contradiction. We are going to 
show that $V_{t}$ and $k_{t}$ have the property that for $y \in p_{2}^{-1}\left(V_{t}\right)$ there exists $x$ such that $\varphi(x)=y$ and

$$
\|x\| \leq \frac{2}{k_{t}}\|y\| .
$$

Now, for $y \in p_{2}^{-1}\left(V_{t}\right), y \neq 0$, there exists $x^{\prime} \in p_{1}^{-1}\left(p_{2}(y)\right)$ such that $\left\|x^{\prime}\right\|<1$ and

$$
\varphi\left(x^{\prime}\right)=\frac{m_{t}}{2\|y\|} y .
$$

We got

$$
\varphi\left(\frac{2\|y\|}{k_{t}} x^{\prime}\right)=y, \quad\left\|\frac{2\|y\|}{k_{t}} x^{\prime}\right\| \leq \frac{2}{k_{t}}\|y\|
$$

The openness of a map between two Banach bundles intervenes also when looking at the preimage of a Banach subbundle. Returning to Example 3.1, one can see that the preimage of the null subbundle $\left\{0_{t} \mid t \in T\right\}$ of $\xi_{2}$ by the map $\varphi$ is $\mathcal{E}^{\prime}:=$ $\{(0,(\mathbb{K} \times\{0\}))\} \cup\{(0,1] \times\{(0,0)\}$ which cannot be the space bundle of a subbundle of $\xi_{1}$. The subset $\left\{(0,(\{\mathcal{R} e(x)>0 \mid x \in \mathbb{K}\} \times\{0\})\}\right.$ is relatively open in $\mathcal{E}^{\prime}$ but its image by the projection $p_{1}$ is $\{0\}$.

Proposition 3.4. Let $\varphi$ be an open Banach bundle map of the Banach bundle $\xi_{1}:=$ $\left(\mathcal{E}_{1}, p_{1}, T\right)$ onto the Banach bundle $\xi_{2}:=\left(\mathcal{E}_{2}, p_{2}, T\right)$ and let $\xi_{2}^{\prime}:=\left(\mathcal{E}_{2}^{\prime}, p_{2} \mid \mathcal{E}_{2}^{\prime}, T\right)$ be a Banach subbundle of $\xi_{2}$. Then, with $\mathcal{E}_{1}^{\prime}:=\varphi^{-1}\left(\mathcal{E}_{2}^{\prime}\right)$, $\left(\mathcal{E}_{1}^{\prime}, p_{1} \mid \mathcal{E}_{1}^{\prime}, T\right)$ is a subbundle of $\xi_{1}$.

Proof. Let $U \subset \mathcal{E}_{1}$ be open and $U \cap \mathcal{E}_{1}^{\prime} \neq \emptyset$. Then $\varphi\left(U \cap E_{1}^{\prime}\right)=\varphi(U) \cap \mathcal{E}_{2}^{\prime}$ is relatively open in $\mathcal{E}_{2}^{\prime}$. It follows that $p_{1}\left(U \cap \mathcal{E}_{1}^{\prime}\right)=p_{2}\left(\varphi(U) \cap \mathcal{E}_{2}^{\prime}\right)$ is open in $T$. We conclude that $p_{1} \mid \mathcal{E}_{1}^{\prime}$ is an open map for the relative topology of $\mathcal{E}_{1}^{\prime}$.

We turn our attention now to the spaces of bounded sections. In order to have $\tilde{\varphi}\left(\Gamma_{b}\left(\xi_{1}\right)\right) \subset \Gamma_{b}\left(\xi_{2}\right)$ for a Banach bundle map between two Banach bundles it is quite natural to require $\sup _{t \in T}\left\|\varphi_{t}\right\|<\infty$. However, even in the situation of Theorem 3.2 one does not necessarily have $\tilde{\varphi}\left(\Gamma_{b}\left(\xi_{1}\right)\right)=\Gamma_{b}\left(\xi_{2}\right)$. Indeed, let $\mathcal{E}_{1}=\mathcal{E}_{2}:=\mathbb{N} \times \mathbb{K}$, $p_{1}=p_{2}$ the projection on $\mathbb{N}$, and $\xi_{i}:=\left(\mathcal{E}_{i}, p_{i}, \mathbb{N}\right), i=1,2, \varphi(n, x):=\left(n, x / n^{2}\right)$. The bounded section of $\xi_{2}$ given by $n \rightarrow(n, 1 / n)$ is the image of only one section of $\xi_{1}$ : the unbounded section $n \rightarrow(n, n)$. 
Theorem 3.5. Let $T$ be a paracompact space and $\xi_{i}:=\left(\mathcal{E}_{i}, p_{i}, T\right), i=1,2$, Banach bundles. Let $\varphi$ be a Banach bundle map of $\xi_{1}$ onto $\xi_{2}$ such that $\sup _{t \in T}\left\|\varphi_{t}\right\|<\infty$. Then $\tilde{\varphi}\left(\Gamma_{b}\left(\xi_{1}\right)\right)=\Gamma_{b}\left(\xi_{2}\right)$ if and only if there is a positive number $m$ with the property that for each $y \in \mathcal{E}_{2}$ there exists $x \in \mathcal{E}_{1}$ such that $\varphi(x)=y$ and $\|x\| \leq m\|y\|$.

Proof. Suppose a constant $m>0$ as in the above statement exists. Then $\varphi$ is an open map by Proposition 3.3 Let $f \in \Gamma_{b}\left(\xi_{2}\right)$ and define the set valued map $\Phi(t):=\left\{x \in p_{1}^{-1}(t) \mid \varphi(x)=f(t),\|x\|<(m+1)\|f\|\right\}, t \in T$. Then $\Phi(t)$ is a non-void convex subset of $p_{1}^{-1}(t)$ for every $t \in T$. We are going to show that $\Phi$ is lower semi-continuous. Let $U$ be an open subset of $\mathcal{E}_{1}$ and suppose $U \cap \Phi\left(t_{0}\right) \neq \emptyset$. Thus there exists $x_{0} \in U \cap\left\{x \in \mathcal{E}_{1} \mid\|x\|<(m+1)\|f\|\right\} \cap \Phi\left(t_{0}\right)$. The subset $\varphi\left(U \cap\left\{x \in \mathcal{E}_{1} \mid\|x\|<(m+1)\|f\|\right\}\right.$ of $\mathcal{E}_{2}$ is an open neighbourhood of $f\left(t_{0}\right)$; hence there exists a neighbourhood $V$ of $t_{0}$ in $T$ such that

$$
f(t) \in \varphi\left(U \cap\left\{x \in \mathcal{E}_{1} \mid\|x\|<(m+1)\|f\|\right\}\right) . t \in V .
$$

Therefore, for each $t \in T$ there exists $x_{t} \in U \cap\left\{x \in \mathcal{E}_{1} \mid\|x\|<(m+1)\|f\|\right\}$ such that $\varphi\left(x_{t}\right)=f(t)$. It follows that $V$ is a neighbourhood of $t_{0}$ such that $U \cap \Phi(t) \neq \emptyset$ for every $t \in V$ and we obtained that $\Phi$ is lower semi-continuous. Now, it is easily seen that $t \rightarrow \overline{\Phi(t)}$ is lower semi-continuous too. Moreover, from $\Phi(t) \subset p_{1}^{-1}(t)$ and the fact that $p_{1}^{-1}(t)$ is closed in $\mathcal{E}_{1}$ we gather that $\|x\| \leq(m+1)\|f\|$ for each $x \in \Phi(t)$. Thus, a continuous selection $g$ of $t \rightarrow \overline{\Phi(t)}$ given by Theorem 2.9 is a bounded section of $\xi_{1}$ that satisfies $\tilde{\varphi}(g)=f$ and we are done with this half of the proof.

Suppose now that $\tilde{\varphi}\left(\Gamma_{b}\left(\xi_{1}\right)=\Gamma_{b}\left(\xi_{2}\right)\right.$. It is a well known consequence of the Banach open mapping theorem that there exists a constant $k>0$ with the property that for every $f \in \Gamma\left(\xi_{2}\right)$ there exists $g \in \Gamma_{b}\left(\xi_{1}\right)$ such that $\tilde{\varphi}(g)=f$ and $\|g\| \leq k\|f\|$. We claim that for every $y \in \mathcal{E}_{2}$ there exists $x \in \mathcal{E}_{1}$ such that $\varphi(x)=y$ and $\|x\| \leq$ $3 k / 2\|y\|$. Indeed, for $y \in E_{2}, y \neq 0$, denote $t:=p_{2}(y)$ and let $f^{\prime} \in \Gamma_{b}\left(\xi_{2}\right)$ satisfy $f^{\prime}(t)=y$. There exists a neighbourhood $V$ of $t$ in $T$ such that $\left\|f^{\prime}\left(t^{\prime}\right)\right\|<3 / 2\left\|f^{\prime}(t)\right\|$ whenever $t^{\prime} \in V$. Let $h: T \rightarrow[0,1]$ be a continuous function such that $h(t)=1$ and $h \mid(T \backslash V) \equiv 0$. Then $f ;=h f^{\prime}$ satisfies $f(t)=y$ and $\|f\| \leq 3 / 2\|y\|$. Let now $g \in \Gamma_{b}\left(\xi_{1}\right)$ be such that $\tilde{\varphi}(g)=f$ and $\|g\| \leq k\|f\|$. Then $\varphi(g(t))=y$ and

$$
\|g(t)\| \leq\|g\| \leq k\|f\| \leq 3 k / 2\|y\|
$$


and this establishes our claim.

Remark 3.6. The 'if' direction of the above theorem in the case of a quotient map is part of [9, Theorem 9.14].

For a Banach bundle $\xi$ whose base space is locally compact Hausdorff the space $\Gamma_{0}(\xi)$ is of interest and we shall look now at the behavior of such spaces under a Banach bundle map.

Lemma 3.7. Let $\xi:=(\mathcal{E}, p, T)$ be a Banach bundle and $h: T \rightarrow(0, \infty)$ a continuous function. Then $\mathcal{F}:=\{x \in \mathcal{E} \mid p(x)=t,\|x\|<h(t)\}$ is an open subset of $\mathcal{E}$.

Proof. Let $x_{0} \in \mathcal{F}$ and put $t_{0}=p\left(x_{0}\right)$. There exists an open neighbourhood $V$ of $t_{0}$ such that $h(t)>h\left(t_{0}\right)-1 / 2\left(h\left(t_{0}\right)-\left\|x_{0}\right\|\right)$ if $t \in V$. Now, it is easily checked that $p^{-1}(V) \cap\left\{x \in \mathcal{E} \mid\|x\|<1 / 2\left(\left\|x_{0}\right\|+h\left(t_{0}\right)\right)\right\} \subset \mathcal{F}$ and we are done.

Theorem 3.8. Let $\xi_{1}:=\left(\mathcal{E}_{1}, p_{1}, T\right)$ be a Banach bundle and $\xi_{2}:=\left(\mathcal{E}_{2}, p_{2}, T\right)$ be a continuous Banach bundle with $T$ locally compact Hausdorff. Suppose that $\varphi$ is a Banach bundle map of $\mathcal{E}_{1}$ onto $\mathcal{E}_{2}$ such that $\sup _{t \in T}\left\|\varphi_{t}\right\|<\infty$. If there exists a positive constant $m$ with the property that for each $y \in \mathcal{E}_{2}$ there exists $x \in \mathcal{E}_{1}$ satisfying $\varphi(x)=y$ and $\|x\|<m\|y\|$ then $\tilde{\varphi}\left(\Gamma_{0}\left(\xi_{1}\right)\right)=\Gamma_{0}\left(\xi_{2}\right)$.

Proof. Let $g \in \Gamma_{0}\left(\xi_{1}\right)$ and $\varepsilon>0$. From

$$
\|\tilde{\varphi}(g)(t)\|=\|\varphi(g(t))\| \leq\|g(t)\| \sup _{t^{\prime} \in T}\left\|\varphi_{t^{\prime}}\right\|
$$

we obtain that $\{t \in T \mid\|\tilde{\varphi}(g)(t)\| \geq \varepsilon\}$ is a closed subset of the compact set $\left\{t \in T \mid\|g(t)\| \geq \varepsilon / \sup _{t^{\prime}}\left\|\varphi_{t^{\prime}}\right\|\right\}$. Thus $\tilde{\varphi}(g) \in \Gamma_{0}\left(\xi_{2}\right)$ and we got $\tilde{\varphi}\left(\Gamma_{0}\left(\xi_{1}\right)\right) \subset$ $\Gamma_{0}\left(\xi_{2}\right)$.

Let now $f \in \Gamma_{0}\left(\xi_{2}\right)$. Then $T^{\prime}:=\{t \in T \mid\|f(t)\|>0\}$ is an open $\sigma$-compact subset of $T$; it is paracompact by [6, Corollary 2, p. 211]. Define

$$
\Phi(t):=\left\{x \in p_{1}^{-1}(t) \mid \varphi(x)=f(t),\|x\|<(m+1)\|f(t)\|\right\}
$$

for $t \in T^{\prime}$. Then $\Phi(t)$ is a non-void convex subset of the Banach space $p_{1}^{-1}(t)$. We are going to show that $t \rightarrow \Phi(t)$ is lower semi-continuous on $T^{\prime}$. With $U$ an open 
subset of $p_{1}^{-1}\left(T^{\prime}\right)$ suppose $U \cap \Phi\left(t_{0}\right) \neq \emptyset, t_{0} \in T^{\prime}$. Let $x_{0} \in U \cap \Phi\left(t_{0}\right)$. Then $\left\|x_{0}\right\|<$ $(m+1)\left\|f\left(t_{0}\right)\right\|$ and $x_{0} \in U \cap\left\{x \in p_{1}^{-1}\left(T^{\prime}\right) \mid\|x\|<1 / 2\left(\left\|x_{0}\right\|+(m+1)\left\|f\left(t_{0}\right)\right\|\right)\right\}$. The set $U^{\prime}:=U \cap\left\{x \in p_{1}^{-1}\left(T^{\prime}\right) \mid\|x\|<1 / 2\left(\left\|x_{0}\right\|+(m+1)\left\|f\left(t_{0}\right)\right\|\right)\right\}$ is an open subset of $\mathcal{E}_{1}$ and $\varphi$ is an open map by Proposition 3.3. hence $\varphi\left(U^{\prime}\right)$ is an open neighbourhood of $\varphi\left(x_{0}\right)=f\left(t_{0}\right)$. There exists an open neighbourhood $V$ of $t_{0}$ in $T^{\prime}$ such that $f(t) \in \varphi\left(U^{\prime}\right)$ and $\|f(t)\|>\left\|f\left(t_{o}\right)\right\|-1 / 2\left(\left\|f\left(t_{0}\right)\right\|-\left\|x_{0}\right\| /(m+1)\right)$ for $t \in V$. Thus, if $t \in V$ there exists $x_{t} \in U^{\prime}$ such that $\varphi\left(x_{t}\right)=f(t)$. Then $\left\|x_{t}\right\|<1 / 2\left(\left\|x_{o}\right\|+(m+1)\left\|f\left(t_{0}\right)\right\|\right)<(m+1)\|f(t)\|$ and we have $x_{t} \in U \cap \Phi(t)$. We got $t_{0} \in V \subset U \cap \Phi(t)$ and we can conclude that $t \rightarrow \Phi(t)$ is indeed lower semi-continuous.

The set valued map $t \rightarrow \overline{\Phi(t)}, t \in T^{\prime}$, is lower semi-continuous too and each set $\overline{\Phi(t)}$ is a non-void closed subset of $\left\{x \in p_{1}^{-1}(t) \mid\|x\| \leq(m+1)\|f(t)\|\right\}$. By Theorem 2.9 there exists a continuous selection $g^{\prime}$ of $\Phi$ on $T^{\prime}$. We have $\varphi\left(g^{\prime}(t)\right)=f(t)$ and $\left\|g^{\prime}(t)\right\| \leq(m+1)\|f(t)\|$ for each $t \in T^{\prime}$. Define now

$$
g(t)= \begin{cases}g^{\prime}(t), & \text { if } t \in T^{\prime}, \\ 0_{t}, & \text { if } t \in T \backslash T^{\prime} .\end{cases}
$$

The function $g: T \rightarrow \mathcal{E}$ is continuous. Indeed, let $\left\{t_{\alpha}\right\}$ be a net in $T^{\prime}$ that converges to $t \in T \backslash T^{\prime}$. then $f\left(t_{\alpha}\right) \rightarrow f(t)=0_{t}$; from $0 \leq\left\|g\left(t_{\alpha}\right)\right\| \leq(m+1)\left\|f\left(t_{\alpha}\right)\right\|$ we derive $\left\|g\left(t_{\alpha}\right)\right\| \rightarrow 0$. Hence $g\left(t_{\alpha}\right) \rightarrow 0_{t}=g(t)$. If $\varepsilon>0$ then $\{t \in T \mid\|g(t)\| \geq \varepsilon\}$ is compact since it is a closed subset of the compact set $\{t \in T \mid\|f(t)\| \geq \varepsilon /(m+1)\}$. We got $g \in \Gamma_{0}\left(\xi_{1}\right)$ with $\tilde{\varphi}(g)=f$ and we conclude $\tilde{\varphi}\left(\Gamma_{0}\left(\xi_{1}\right)\right)=\Gamma_{0}\left(\xi_{2}\right)$.

We shall now consider results suggested by [3] and [17] on the existence of right inverses for some Banach bundle maps.

Proposition 3.9. Let $\varphi$ be an open Banach bundle map of $\xi_{1}:=\left(\mathcal{E}_{1}, p_{1}, T\right)$ onto $\xi_{2}:=\left(\mathcal{E}_{2}, p_{2}, T\right)$. Suppose that $\mathcal{E}_{2}$ is paracompact. Then there exists a continuous map $\psi: \mathcal{E}_{2} \rightarrow \mathcal{E}_{1}$ such that $\varphi(\psi(y))=$ y for every $y \in \mathcal{E}_{2}$.

Proof. Define $\Phi(y):=\varphi^{-1}(y), y \in \mathcal{E}_{2}$. By using the fact that $\varphi$ is open one easily sees that $\Phi$ is lower semi-continuous. An application of Theorem 2.9 in the obvious manner yields a continuous selection $\psi: \mathcal{E}_{2} \rightarrow \mathcal{E}_{1}$ of $\Phi$ that fulfills what is needed. 
To obtain a homogeneous right inverse we shall suppose that the range of the Banach bundle map is a continuous Banach bundle. But first we have to state two simple lemmas. We omit their elementary proofs; the first can be proven by the means of an obvious compactness argument and the second lemma is an easy consequence of the first. We let $\mu$ be the normalized Lebesgue measure on the unit circle in $\mathbb{C}$ and $\widehat{\lambda_{1} \lambda_{2}}$ will denote the arc of the unit circle from $\lambda_{1}$ to $\lambda_{2}$ in the positive direction.

Lemma 3.10. Let $\xi_{i}:=\left(\mathcal{E}_{i}, p_{i}, T\right), i=1,2$ be two Banach bundles over the complex field, $f: \mathcal{E}_{2} \rightarrow \mathcal{E}_{1}$ a continuous function and $\left\{y_{\alpha}\right\}_{\alpha \in \mathcal{A}}$ a net in $\mathcal{E}_{2}$ that converges to $y \in \mathcal{E}_{2}$. Given $\varepsilon>0$ there exists $\delta>0$ such that $\left|f\left(\lambda_{1} y_{\alpha}\right)-f\left(\lambda_{2} y_{\alpha}\right)\right|<\varepsilon$ and $\left|f\left(\lambda_{1} y\right)-f\left(\lambda_{2} y\right)\right|<\varepsilon$ whenever $\left|\lambda_{1}\right|=\left|\lambda_{2}\right|=1, \mu\left(\widehat{\lambda_{1} \lambda_{2}}\right)<\delta$ and for every $\alpha \in \mathcal{A}$.

Lemma 3.11. Let $\xi_{1}, \xi_{2}, f,\left\{y_{\alpha}\right\}_{\alpha \in \mathcal{A}}$, and $y$ be as in Lemma 3.10. Given $\varepsilon>0$ there exists $\delta>0$ with the following property: if $\left\{\lambda_{i}\right\}_{i=1}^{n}, \lambda_{1}=\lambda_{n}$, is a division of the unit circle in the complex plane with mesh smaller than $\delta$ then

$$
\left|\int_{|\lambda|=1} f(\lambda y) d \mu(\lambda)-\sum_{i=1}^{n-1} f\left(\lambda_{i} y\right) \mu\left(\widehat{\lambda_{i} \lambda_{i+1}}\right)\right|<\varepsilon
$$

and

$$
\left|\int_{|\lambda|=1} f\left(\lambda y_{\alpha}\right) d \mu(\lambda)-\sum_{i=1}^{n-1} f\left(\lambda_{i} y_{\alpha}\right) \mu\left(\widehat{\lambda_{i} \lambda_{i+1}}\right)\right|<\varepsilon
$$

for every $\alpha \in \mathcal{A}$.

Proposition 3.12. Let $\varphi$ be a Banach bundle map from the Banach bundle $\xi_{1}:=$ $\left(\mathcal{E}_{1}, p_{1}, T\right)$ onto the continuous Banach bundle $\xi_{2}:=\left(\mathcal{E}_{2}, p_{2}, T\right)$. Suppose that $\mathcal{E}_{2}$ is paracompact and there exists $m>0$ with the propriety that for every $y \in \mathcal{E}_{2}$ there exists $x \in \mathcal{E}_{1}$ such that $\varphi(x)=y$ and $\|x\| \leq m\|y\|$. Then there exists a continuous map $\psi:=\mathcal{E}_{2} \rightarrow \mathcal{E}_{1}$ such that $\varphi(\psi(y))=y$ and $\psi(\lambda y)=\lambda \psi(y)$ for every $y \in \mathcal{E}_{2}$ and $\lambda \in \mathbb{K}$.

Proof. The set $S:=\left\{y \in \mathcal{E}_{2} \mid\|y\|=1\right\}$ is closed in $\mathcal{E}_{2}$ thus paracompact. We define $\Phi(y):=\left\{x \in \mathcal{E}_{1} \mid \varphi(x)=y,\|x\|<m+1\right\}, y \in S$. Then $\Phi(y)$ is a non-empty convex subset of $p_{1}^{-1}\left(p_{2}(y)\right)$ and we want to show that $\Phi$ is lower semicontinuous. So let $U$ be an open subset of $\mathcal{E}_{1}$ and suppose $U \cap \Phi\left(y_{0}\right) \neq \emptyset$. Then 
$U^{\prime}:=\varphi\left(U \cap\left\{x \in \mathcal{E}_{1} \mid\|x\|<m+1\right\}\right)$ is an open subset of $\mathcal{E}_{2}$ since $\varphi$ is an open map by Proposition 3.3 and $y_{0} \in U^{\prime} \cap F$. If $y \in U^{\prime} \cap F$ then there exists $x \in U$ such that $\|x\|<m+1$ and $\varphi(x)=y$. Thus $U \cap \Phi(y) \neq \emptyset$ if $y \in U^{\prime} \cap F$ and we have proved that $\Phi$ is lower semi-continuous. The map $y \rightarrow \overline{\Phi(y)}$ is also lower semi-continuous and Theorem 2.9 yields a continuous function $g: F \rightarrow \mathcal{E}_{1}$ such that $\varphi(g(y))=y$ and $\|g(y)\| \leq m+1$ for $y \in F$. By defining

$$
h(y):= \begin{cases}\|y\| g(y /\|y\|), & \text { if }\|y\| \neq 0, \\ 0, & \text { otherwise }\end{cases}
$$

for $y \in \mathcal{E}_{2}$ one gets a continuous function from $\mathcal{E}_{2}$ to $\mathcal{E}_{1}$ such that $\varphi(h(y))=y$ for $y \in \mathcal{E}_{2} .$. If the scalars are real then one easily checks that $\psi(y):=1 / 2(h(y)-h(-y))$ has the required properties. Suppose now that the scalars are complex. Then we define

$$
\psi(y):=\int_{|\lambda|=1} \bar{\lambda} h(\lambda y) d \mu(\lambda), \quad y \in \mathcal{E}_{2} .
$$

The function $\psi$ has all the needed properties. We shall prove only its continuity all its other attributes being straightforward to check. So let $y \in \mathcal{E}_{2}$ and suppose $\left\{y_{\alpha}\right\}_{\alpha \in \mathcal{A}}$ is a net that converges to $y$. We denote $t:=p_{2}(y)$ and $t_{\alpha}:=p_{2}\left(y_{\alpha}\right)$. Let $U$ be an open neighbourhood of $\psi(y)$. Now $T$ is paracompact being homeomorphic to the closed subspace $\left\{0_{\tau}\right\}_{\tau \in T}$ of $\mathcal{E}_{2}$. So there exist a section $\sigma$ of $\xi_{1}$, a neighbourhood $V$ of $t$ contained in $p_{1}(U)$ and $\varepsilon>0$ such that $\sigma(t)=\psi(y)$ and

$$
U^{\prime}:=\left\{x \in \mathcal{E}_{1} \mid p_{1}(x) \in V, \quad\left\|x-\sigma\left(p_{1}(x)\right)\right\|<2 \varepsilon\right\} \subset U .
$$

Let now $\delta$ be the positive number given by Lemma 3.11 for our number $\varepsilon$ and the integrant appearing in the definition of $\psi$. Suppose $\left\{\lambda_{i}\right\}_{i=1}^{n}, \lambda_{1}=\lambda_{n}$, is a division of the unit circle of mesh less than $\delta$. Then $\left\|\psi(y)-\sum_{i=1}^{n-1} \bar{\lambda}_{i} h\left(\lambda_{i} y\right) \mu\left(\widehat{\lambda_{1} \lambda_{i+1}}\right)\right\|<\varepsilon$. We denote

$$
U^{\prime \prime}:=\left\{x \in \mathcal{E}_{1} \mid p_{1}(x) \in V, \quad\left\|x-\sigma\left(p_{1}(x)\right)\right\|<\varepsilon\right\} ;
$$

then $\sum_{i=1}^{n-1} \bar{\lambda}_{i} h\left(\lambda_{i} y\right) \mu\left(\widehat{\lambda_{i} \lambda_{i+1}}\right)$ belongs to the open set $U^{\prime \prime}$. There exists $\alpha_{0} \in \mathcal{A}$ such that if $\alpha \succ \alpha_{0}$ then $\sum_{i=1}^{n-1} \bar{\lambda}_{i} h\left(\lambda_{i} y_{\alpha}\right) \mu\left(\widehat{\lambda_{i} \lambda_{i+1}}\right)$ also belongs to $U^{\prime \prime}$. Thus we have $\left\|\sum_{i=1}^{n-1} \bar{\lambda}_{i} h\left(\lambda_{i} y_{\alpha}\right) \mu\left(\widehat{\lambda_{i} \lambda_{i+1}}\right)-\sigma\left(p_{2}\left(y_{\alpha}\right)\right)\right\|<\varepsilon$ if $\alpha \succ \alpha_{0}$ and

$$
\left\|\psi\left(y_{\alpha}\right)-\sum_{i=1}^{n-1} \bar{\lambda}_{i} h\left(\lambda_{i} y_{\alpha}\right) \mu\left(\widehat{\lambda_{i} \lambda_{i+1}}\right)\right\|<\varepsilon .
$$

We obtained that $\psi\left(y_{\alpha}\right) \in U^{\prime} \subset U$ if $\alpha$ is large enough so we conclude that $\left\{\psi\left(y_{\alpha}\right)\right\}$ converges to $\psi(y)$. 
Remark 3.13. It follows from the proof of Proposition 3.12 that the map $\psi$ satisfies $\|\psi(y)\| \leq(m+1)\|y\|$ for every $y \in \mathcal{E}_{2}$. Thus one can easily obtain a proof of Theorem 3.8 by using Proposition 3.12 if in addition to the other hypotheses of that theorem one supposes that $\mathcal{E}_{2}$ is paracompact.

\section{M-IDEALS OF $\Gamma_{0}(\xi)$}

For the present section $\xi:=(\mathcal{E}, p, T)$ will be a fixed Banach bundle. We shall mainly discuss the M-ideals of the Banach space $\Gamma_{0}(\xi)$ when the base space is locally compact Hausdorff; most of the results that follow are generalizations of results from 9] where only the case of a compact Hausdorff base space was considered. As in [9], for a closed subset $A$ of $T$ the notation $N_{A}$ will stand for $\left\{\sigma \in \Gamma_{b} \mid \sigma(t)=\right.$ $0_{t} \quad$ for all $\left.t \in A\right\}$ or for $\left\{\sigma \in \Gamma_{0}(\xi) \mid \sigma(t)=0_{t}\right.$ for all $\left.t \in A\right\}$ if $T$ is locally compact Hausdorff; it will be clear from the context which is the case. If $A$ is a singleton, say $\{t\}$, then we shall use the notation $N_{t}$ instead of $N_{\{t\}}$.

In [9, Proposition 13.6] the base space is assumed to be compact Hausdorff. However the proof given there is valid with obvious modifications in the more general situations detailed in the next Proposition. Of course, we omit its proof.

Proposition 4.1. The closed subspace $N_{A}$ is an $M$-ideal of $\Gamma_{b}(\xi)$ if $A$ is a closed subset of the normal space $T$. For a locally compact Hausdorff base space $T N_{A}$ is an $M$-deal of $\Gamma_{0}(\xi)$ if $A$ is a compact subset of $T$ or if $A$ is a closed subset of $T$ such that the complement of its interior is compact.

Proposition 4.2. Suppose $T$ is paracompact (locally compact Hausdorff) and $A$ and $B$ are closed (compact, respectively) subsets of $T$. Then $N_{A \cap B}=N_{A}+N_{B}$.

Proof. We must prove only $N_{A \cap B} \subset N_{A}+N_{B}$. Let $\sigma \in N_{A \cap B}$ and define $\rho$ : $A \cup B \rightarrow \mathcal{E}$ by $\rho(t):=\sigma(t)$ if $t \in A$ and $\rho(t):=0_{t}$ if $t \in B$. Then $\rho$ is well defined and continuous on $A \cup B$. Corollary 2.10 provides us with an extension of $\varrho$ in $\Gamma_{b}(\xi)\left(\Gamma_{0}(\xi)\right.$, respectively); we shall call this extension $\rho_{B}$. We have $\rho_{B} \in N_{B}$ and $\rho_{A}:=\sigma-\rho_{B}$ is in $A$. 
Proposition 4.2 appeared as [9, Corollary 15.8] for $T$ compact Hausdorff and was given there a different proof from the one above.

An M-ideal $L$ of a Banach space $X$ is called primitive if there is an extreme point $\omega$ of the closed unit ball of the dual space $X^{\prime}$ such that $L$ is maximal among the M-ideals contained in the null subspace of $\omega$. If $L$ is a primitive M-ideal and $L_{1}$, $L_{2}$ are M-ideals such that $L_{1} \cap L_{2} \subset L$ then $L_{1} \subset L$ or $L_{2} \subset L$. Every M-ideal is the intersection of the primitive M-ideals containing it. For all these facts see [1, Section 3].

In the remainder of this section the base space $T$ will always be a locally compact Hausdorff space.

The case $T$ compact Hausdorff of the following proposition is part of $[9$, Proposition 13.11].

Proposition 4.3. If $L$ is a primitive $M$-ideal of $\Gamma_{0}(\xi)$ then there is a unique $t_{0} \in T$ such that $N_{t_{0}} \subset L$.

Proof. Let $K$ be a non-void compact subset of $T$. Then $N_{K}$ and $N_{\overline{T \backslash K}}$ are Mideals of $\Gamma_{0}(\xi)$ by Proposition 4.1 and $N_{K} \cap N_{\overline{T \backslash K}}=\{0\} \subset L$. We infer that $N_{K}$ or $N_{\overline{T \subset K}}$ is included in $L$. If for every such $K$ we have $N_{\overline{T \backslash K}} \subset L$ then $L=\Gamma_{0}(\xi)$ since the sections with compact support are dense in $\Gamma_{0}(\xi)$. Thus there exists a non-void compact set $K$ such that $N_{K} \subset L$. Let $\mathcal{F}$ be the non-void family of all non-void compact subsets of $T$ such that $N_{K} \subset L$. If $K_{1}$ and $K_{2}$ belong to $\mathcal{F}$ then $N_{K_{1} \cap K_{2}}=N_{K_{1}}+N_{K_{2}} \subset L$ and it follows that $\mathcal{F}$ has the finite intersection property. Hence $K_{0}:=\cap\{K \mid K \in \mathcal{F}\} \neq \emptyset$.

Denote

$$
\Lambda:=\overline{\bigcup_{K \in \mathcal{F}} N_{K}}
$$

We claim that $N_{K_{0}}=\Lambda$. Obviously $\Lambda \subset N_{K_{0}}$. Let now $\sigma \in N_{K_{0}}$ and $\varepsilon>0$. Set $V:=\{t \in T \mid\|\sigma(t)\|<\varepsilon\}$; then $V$ is open and $K_{0} \subset V$. If it were true that for each $K \in \mathcal{F}$ there would be $t_{K} \in K \backslash V$ then the net $\left\{t_{K}\right\}_{K \in \mathcal{F}}$ would have a convergent subnet whose limit would be in $K_{0}$. On the other hand, $\left\|\sigma\left(t_{k}\right)\right\| \geq \varepsilon$ for all $K \in \mathcal{F}$, a contradiction. Thus there exists $K \in \mathcal{F}$ such that $K \subset V$. Let $f: T \rightarrow[0,1]$ be a continuous function such that $f \mid K \equiv 0$ and $f \mid(T \backslash V) \equiv 1$. Then with $\sigma^{\prime}:=f \cdot \sigma$ we have $\sigma^{\prime} \in N_{K}$ and $\left\|\sigma-\sigma^{\prime}\right\|<\varepsilon$. We infer that $\sigma \in \Lambda$ and the claim follows from this. In particular, $K_{0} \in \mathcal{F}$. 
It remains only to show that $K_{0}$ consists of only one point. If there are in $K_{0}$ at least two distinct points then there exist compact subsets $K_{1}, K_{2}$ of $K_{0}$ such that $K_{0}=K_{1} \cup K_{2}$ and $K_{1} \neq K_{0} \neq K_{2}$. These subsets will have to satisfy $N_{K_{1}} \cap N_{K_{2}}=N_{K_{0}} \subset L$. Hence $N_{K_{1}} \subset L$ or $N_{K_{2}} \subset L$. But $K_{i} \in \mathcal{F}, i=1$ or 2 , and $K_{i} \varsubsetneqq K_{0}$ is a contradiction. Therefore $K_{0}=\left\{t_{0}\right\}$ for some $t_{0} \in T$ and we found $N_{t_{0}} \subset L$.

The uniqueness of $t_{0}$ with the property $N_{t_{0}} \subset L$ follows from the definitions of $\mathcal{F}$ and $K_{0}$.

Now we can apply the same proof as in the compact case of [9, Proposition 15.20] to get

Proposition 4.4. Every $M$-ideal $L$ of $\Gamma_{0}(\xi)$ is a $C_{b}(T)$-module.

Proof. Suppose first that $L$ is a primitive M-ideal and let $t_{0} \in T$ be the element given by Proposition 4.3. If $f \in C_{b}(T)$ and $\sigma \in L$ then $f \cdot \sigma-f\left(t_{0}\right) \sigma$ belongs to $N_{t_{0}} \subset L$. Hence $f \cdot \sigma \in L$ and the case of a primitive M-ideal is settled. The general case follows from this particular case and the fact mentioned above that each M-ideal is the intersection of the primitive M-ideals containing it.

We proceed now to give a characterization of those closed subspaces of $\Gamma_{0}(\xi)$ that are $C_{b}(T)$-modules. The parallel result for paracompact base spaces and spaces of bounded sections is [9, Theorem 8.6]. First we state and prove a simple lemma.

Lemma 4.5. Let $L$ be an $M$-ideal of $\Gamma_{0}(\xi), \sigma \in L$, and suppose that $\left\|\sigma\left(t_{0}\right)\right\|<\theta$ for some $t_{0} \in T$ and $\theta>0$. Then there is $\sigma^{\prime} \in L$ such that $\sigma^{\prime}\left(t_{0}\right)=\sigma\left(t_{0}\right)$ and $\left\|\sigma^{\prime}\right\|<\theta$.

Proof. Denote $\theta^{\prime}:=1 / 2\left(\theta+\left\|\sigma\left(t_{0}\right)\right\|\right)$. Then $V:=\left\{t \in T \mid\|\sigma(t)\|<\theta^{\prime}\right\}$ is an open neighbourhood of $t_{0}$ and $T \backslash V$ is compact. Let $f: T \rightarrow[0,1]$ be a continuous function such that $f\left(t_{0}\right)=1$ and $f \mid(T \backslash V) \equiv 0$. Define $\sigma^{\prime}:=f \cdot \sigma$. It is easily checked that $\sigma^{\prime}$ has the required properties. 
Theorem 4.6. A closed subspace of $\Gamma_{0}(\xi)$ is a $C_{b}(T)$-submodule if and only if there exists a subbundle $\eta$ of $\xi$ such that $L=\Gamma_{0}(\eta)$.

Proof. Obviously $\Gamma_{0}(\eta)$ for $\eta$ a subbundle of $\xi$ is a $C_{b}(T)$-submodule of $\Gamma_{0}(\xi)$. Suppose now that the closed subspace $L$ of $\Gamma_{0}(\xi)$ is a $C_{b}(T)$-submodule. Denote $\mathcal{F}(t):=\{\sigma(t) \mid \sigma \in L\}, t \in T$. Clearly $\mathcal{F}(t)$ is a linear subspace of $\mathcal{E}(t)$ and we are going to show that it is closed in $\mathcal{E}(t)$. Let $\left\{x_{n}\right\}$ be a sequence in $\mathcal{F}(t)$ that converges to $x \in \mathcal{E}(t)$. By passing to a subsequence if necessary we may suppose that $\left\|x_{n+1}-x_{n}\right\|<2^{-n}$ for each $n \in \mathbb{N}$. There exist $\sigma_{n} \in L$ such that $\sigma_{n}(t)=x_{n}$, $n \in \mathbb{N}$. Put $\varrho_{1}:=\sigma_{1} ;$ Lemma 4.5 yields $\varrho_{n} \in L$ with $\varrho_{n}(t)=\sigma_{n+1}(t)-\sigma_{n}(t)$ and $\left\|\varrho_{n}\right\|<2^{-n}, n \geq 2$. The section $\varrho ;=\sum_{n=1}^{\infty} \varrho_{n}$ is in $L$ and $\varrho(t)=\lim \sigma_{n}(t)=x$ and we deduce that $x \in \mathcal{F}(t)$.

Set $\mathcal{F}:=\cup_{t \in T} \mathcal{F}(t)$; we are going to show that $p \mid \mathcal{F}$ is open. Let $U$ be an open subset of $\mathcal{E}$ and $t_{0} \in p(U \cap \mathcal{F})$. There exists $\sigma \in L$ such that $\sigma\left(t_{0}\right) \in U \cap \mathcal{F}$. there exists a neighbourhood $V$ of $t_{0}$ in $T$ such that $\sigma(t) \in U$ whenever $t \in V$. Then $V \subset p(U \cap \mathcal{F})$ and we obtained that $p(U \cap \mathcal{F})$ is an open subset of $T$. We have shown that $\eta:=(\mathcal{F}, p \mid \mathcal{F}, T)$ is a subbundle of $\xi$.

We want to show now that $L=\Gamma_{0}(\eta)$. Clearly $L \subset \Gamma_{0}(\eta)$. Let $\sigma \in \Gamma_{0}(\eta)$ and $\varepsilon>0$. Then $K:=\{t \in T \mid\|\sigma(t)\| \geq \varepsilon\}$ is a compact subset of $T$ and, $L$ being a $C_{b}(T)$ module, for each $t \in K$ there exists $\varrho_{t} \in L$ such that $\varrho_{t}(t)=\sigma(t)$ and $\varrho_{t} \mid(T \backslash K) \equiv 0$. The set $V_{t}:=\left\{t^{\prime} \in T \mid\left\|\sigma\left(t^{\prime}\right)-\varrho_{t}\left(t^{\prime}\right)\right\|<\varepsilon\right\}, t \in K$, is open; for each $t \in K$ we choose on open set $W_{t}$ such that $\overline{W_{t}}$ is compact and $t \in W_{t} \subset \overline{W_{t}} \subset V_{t}$. There is a finite subcover $\left\{W_{t_{i}}\right\}_{i=1}^{n}$ of the cover $\left\{W_{t}\right\}_{t \in K}$ of $K$; let $f_{i}: T \rightarrow[0,1]$ be a continuous function such that $f_{i} \mid \overline{W_{t_{i}}} \equiv 1$ and $f_{i} \mid\left(T \backslash V_{t_{i}}\right) \equiv 0,1 \leq i \leq n$. On $\cup_{i=1}^{n} \overline{W_{t_{i}}}$ set

$$
g_{i}:=\frac{f_{i}}{\sum_{j=1}^{n} f_{j}}
$$

and extend each $g_{i}$ to a continuous and bounded function on $T$. For simplicity, the extension will be denoted also by $g_{i}$. Define now $\rho:=\sum_{i=1}^{n} g_{i} \varrho_{t_{i}}$. Then $\rho \in L$ and for $t \in K$ we have

$$
\|\rho(t)-\sigma(t)\| \leq \sum_{i=1}^{n} g_{i}\left\|\varrho_{t_{i}}-\sigma(t)\right\|<\varepsilon
$$

Define the open neighbourhood $V:=\{\|\rho(t)-\sigma(t)\|<\varepsilon$ of $K$ and let $h: T \rightarrow[0,1]$ be a continuous function such that $h \mid K \equiv 1$ and $h \mid(T \backslash V) \equiv 0$. Then $h \cdot \rho \in L$ 
and $\|h(t) \rho(t)-\sigma(t)\|<\varepsilon$ on $K$. If $t \in T \backslash V$ then $\|h(t) \rho(t)-\sigma(t)\|=\|\sigma(t)\|<\varepsilon$ since $K \subset V$. Now, if $t \in V \backslash K$ we have

$$
\begin{aligned}
\|h(t) \rho(t)-\sigma(t)\| \leq & |h(t)-1|\|\rho(t)\|+\|\rho(t)-\sigma(t)\| \leq \\
& (1-h(t))(\|\rho(t)-\sigma(t)\|+\|\sigma(t)\|)+\|\rho(t)-\sigma(t)\|<3 \varepsilon
\end{aligned}
$$

We have found $h \cdot \rho \in L$ such that $\|h \cdot \rho-\sigma\| \leq 3 \varepsilon$ hence $\sigma \in L$ and we conclude that $L=\Gamma_{0}(\eta)$.

The case of a compact base space of the following result is [9, Theorem 15.21] for which only a brief indication of the proof was given.

Theorem 4.7. A closed subspace $L$ of $\Gamma_{0}(\xi)$ is an $M$-ideal if and only if there exists a subbundle $\eta:=(\mathcal{F}, p \mid \mathcal{F}, t)$ of $\xi$ such that $L=\Gamma_{0}(\eta)$ and and each fiber $\mathcal{F}(t)$ is an $M$-deal of the Banach space $\mathcal{E}(t)$.

Proof. Suppose first that $L$ is an M-ideal of $\Gamma(\xi)$. It follows from Proposition 4.4 and Theorem 4.6 that there exists a subbundle $\eta:=(\mathcal{F}, p \mid \mathcal{F}, T)$ of $\xi$ such that $L=\Gamma_{0}(\eta)$. It remains to show that $\mathcal{F}(t)=\{\sigma(t) \mid \sigma \in L\}$ is an M-ideal of $\mathcal{E}(t)$. For this purpose we proceed to show that $\mathcal{F}\left(t_{0}\right)$ has the 3-ball-property in $\mathcal{E}\left(t_{0}\right)$, $t_{0} \in T$. Let $\left\{x_{i}\right\}_{i=1}^{3} \subset \mathcal{E}\left(t_{0}\right)$ and let $\left\{r_{i}\right\}_{i=1}^{3}$ be positive numbers such that there exists $x \in \mathcal{E}\left(t_{0}\right)$ with $\left\|x_{i}-x\right\|<r_{i}, 1 \leq i \leq 3$, and there exist $\left\{y_{i}\right\}_{i=1}^{3}$ in $\mathcal{F}\left(t_{0}\right)$ such that $\left\|x_{i}-y_{i}\right\|<r_{i}, 1 \leq i \leq 3$. There are sections $\sigma, \sigma_{i} \in \Gamma_{0}(\xi)$, such that $\sigma\left(t_{0}\right)=x$ and $\sigma_{i}\left(t_{0}\right)=x_{i}, 1 \leq i \leq 3$, and sections $\varrho_{i} \in \Gamma_{0}(\eta)$ such that $\varrho_{i}\left(t_{0}\right)=y_{i}$, $1 \leq i \leq 3$. The set

$$
V ;=\left\{t \in T \mid\left\|\sigma_{i}(t)-\sigma(t)\right\|<r_{i},\left\|\sigma_{i}(t)-\varrho_{i}(t)\right\|<r_{i}, 1 \leq i \leq 3\right\}
$$

is an open neighbourhood of $t_{0}$. Let $f: T \rightarrow[0,1]$ be a continuous function such that $f\left(t_{0}\right)=1$ and $f \mid(T \backslash V) \equiv 0$. Then $f \cdot \sigma, f \cdot \sigma_{i} \in \Gamma_{0}(\xi)$ and $f \cdot \varrho_{i} \in \Gamma_{0}(\eta)$, $1 \leq i \leq 3$. We have $\left\|f \cdot \sigma_{i}-f \cdot \sigma\right\|<r_{i}$ and $\left\|f \cdot \sigma_{i}-f \cdot \varrho_{i}\right\|<r_{i}, 1 \leq i \leq 3$. Since $\Gamma_{0}(\eta)$ is an M-ideal there is a section $\varrho \in \Gamma_{0}(\eta)$ such that $\left\|f \cdot \sigma_{i}-\varrho\right\|<r_{i}$, $1 \leq i \leq 3$. Then $\varrho\left(t_{0}\right) \in \mathcal{F}\left(t_{0}\right)$ and $\left\|x_{i}-\varrho\left(t_{0}\right)\right\|=\left\|f\left(t_{0}\right) \sigma_{i}\left(t_{0}\right)-\varrho_{i}\left(t_{0}\right)\right\|<r_{i}$, $1 \leq i \leq 3$. Thus $\mathcal{F}\left(t_{0}\right)$ has indeed the 3 -ball-property in $\mathcal{E}\left(t_{0}\right)$.

Now we suppose that $\eta:=(\mathcal{F}, p \mid \mathcal{F}, t)$ is a subbundle of $\xi$ such that each $\mathcal{F}(t)$ is an M-ideal of $\mathcal{E}(t)$. Since each subspace $\mathcal{F}(t)$ is closed in $\mathcal{E}(t)$ it is obvious that 
$\Gamma_{0}(\eta)$ is a closed subspace of $\Gamma_{0}(\xi)$. We are going to show that $\Gamma_{0}(\eta)$ has the 3 -ballproperty in $\Gamma_{0}(\xi)$ and is thus an M-ideal of $\left.\Gamma_{0} \xi\right)$. Let $\left\{r_{i}\right\}_{i=1}^{3}$ be positive numbers, $\sigma_{i}, \sigma \in \Gamma_{0}(\xi)$, and $\varrho_{i} \in \Gamma_{0}(\eta), 1 \leq i \leq 3$, so that $\left\|\sigma_{i}-\sigma\right\|<r_{i}$ and $\left\|\sigma_{i}-\varrho_{i}\right\|<r_{i}$, $1 \leq i \leq 3$. Let $r_{i}^{\prime}$ be a number satisfying

$$
\max \left\{\left\|\sigma_{i}-\sigma\right\|,\left\|\sigma_{i}-\varrho_{i}\right\|\right\}<r_{i}^{\prime}<r_{i},
$$

$1 \leq i \leq 3$ and set

$$
C(t):=\cap_{i=1}^{3}\left\{y \in \mathcal{F}(t) \mid\left\|\sigma_{i}(t)-y\right\|<r_{i}^{\prime}\right\} .
$$

The subspace $\mathcal{F}(t)$ of $\mathcal{E}(t)$ has the 3 -ball-property; hence $C(t)$ is a non-void convex subset of $\mathcal{F}(t), t \in T$. We are going to show that the map $t \rightarrow C(t)$ is lower semi-continuous. Let $U$ be an open subset of $\mathcal{E}$ and suppose $C\left(t_{0}\right) \cap U \neq \emptyset$ for some $t_{0} \in T$. Then there exists an element $y_{0} \in C\left(t_{0}\right) \cap U$ and $\varrho_{0} \in \Gamma_{0}(\eta)$ such that $\varrho_{0}\left(t_{0}\right)=y_{0}$. The open neighbourhood

$$
V:=\left(\cap_{i=1}^{3}\left\{t \in T \mid\left\|\sigma_{i}\left(t_{0}\right)-\varrho_{0}\left(t_{0}\right)\right\|<r_{i}^{\prime}\right\}\right) \cap\{t \in T \mid \varrho(t) \in U\}
$$

of $t_{0}$ satisfies $V \subset\{t \in T \mid C(t) \cap U \neq \emptyset\}$ and the claim about $t \rightarrow C(t)$ is proved. We infer that $t \rightarrow \overline{C(t)}, t \in T$, is also lower semi-continuous. Remark that $\overline{C(t)}$ is a non-void closed convex subset of $\mathcal{F}(t)$. Moreover, if $y \in$ overline $C(t)$ then $\|y\| \leq r_{i}^{\prime}$ The set

$$
K_{1}:=\cup_{i=1}^{3}\left\{t \in T \mid\left\|\sigma_{i}(t)\right\| \geq r_{i}^{\prime} / 2\right\}
$$

is compact and there exists an open subset $U \supset K_{1}$ of $T$ such that $K_{2}:+\bar{U}$ is compact. Let $\rho^{\prime}$ be a continuous selection of $t \rightarrow \overline{C(t)}$ over $K_{2}$ given by Theorem 2.9 and $f: T \rightarrow[0,1]$ be a continuous function satisfying $f \mid K_{1} \equiv 1$ and $f \mid(T \backslash U) \equiv 0$. Define

$$
\rho(t):= \begin{cases}f(t) \cdot \rho^{\prime}(t), & \text { if } t \in K_{2}, \\ 0_{t}, & \text { if } t \in T \backslash U .\end{cases}
$$

Then $\rho$ is well defined and $\rho_{1} \Gamma_{0}(\eta)$. We claim that $\left\|\sigma_{i}-\rho\right\| \leq r_{i}^{\prime}, 1 \leq i \leq 3$ and we shall conclude from this that $\Gamma_{0}(\eta)$ has the 3 -ball-property in $\Gamma_{o}(\xi)$. If $t \in K_{1}$ then $\left\|\sigma_{i}(t)-\rho(t)\right\|=\left\|\sigma_{i}(t)-\rho^{\prime}(t)\right\| \leq r_{i}^{\prime}$ since $\rho^{\prime}(t) \in \overline{C(t)}$. If $t \in T \backslash U$ then $\left\|\sigma_{i}(t)-\rho(t)\right\|=\left\|\sigma_{i}(t)\right\|<r_{i}^{\prime} / 2$. Finally, if $t \in U \backslash K_{1}$ then

$$
\begin{aligned}
& \left\|\sigma_{i}(t)-\rho(t)\right\| \leq\left\|\sigma_{i}(t)-f(t) \cdot \sigma_{i}(t)\right\|+\left\|f(t) \cdot \sigma_{i}(t)-f(t) \cdot \rho^{\prime}(t)\right\| \\
& \quad(1-f(t))\left\|\sigma_{i}(t)\right\|+f(t)\left\|\sigma_{i}(t)-\rho^{\prime}(t)\right\| \leq(1-f(t)) r_{i}^{\prime} / 2+f(t) r_{i}^{\prime} \leq r_{i}^{\prime},
\end{aligned}
$$

and with this the claim is proved. 


\section{UNIFORM AND LOCALLY UNIFORM BANACH BUNDLES}

In this section we shall describe a class of Banach bundles that is more general than the class of locally trivial Banach bundles but it is still quite manageable. This class was introduced in [15] under the guise of continuous fields of Banach spaces. We remind the definition of the Hausdorff distance between two bounded subsets $A, B$ of a metric space $(M, \rho)$ :

$$
d(A, B):=\max \left\{\sup _{a \in A} \rho(a, B), \sup _{b \in B} \rho(b, A)\right\} .
$$

It is a metric in the family of all bounded closed subsets of $M$.

Recall that if $X$ is a Banach space then $X_{1}$ denotes its closed unit ball.

Lemma 5.1. Let $X$ be a Banach space, $\mathcal{M}$ the family of its bounded closed subsets endowed with the Hausdorff metric, $T$ a topological space, and $t \rightarrow X(t)$ a map $\Phi$ from $T$ to the collection of closed subspaces of $X$. If the map $t \rightarrow X(t)_{1}$ from $T$ to $\mathcal{M}$ is continuous then $\Phi$ is lower semi-continuous.

Proof. Let $O$ be an open subset of $X$ and suppose that $O \cap X\left(t_{o}\right) \neq \emptyset, t_{0} \in T$. Pick $x_{0} \in O \cap X\left(t_{0}\right)$ and let $r>0$ be so that $B\left(x_{0}, r\right) \subset O$. From the continuity of $t \rightarrow X(t)_{1}$ it follows that there exists a neighbouhood $V$ of $t_{0}$ in $T$ such that for each $t \in V$ there exists $y_{t} \in X(t)$ satisfying $\left\|y_{t}\right\| \leq\left\|x_{0}\right\|$ and $\left\|x_{0}-y_{t}\right\|<r$. Thus, if $t \in V$ then $O \cap X(t) \neq \emptyset$ and the proof is complete.

We keep the setting and the notations of Lemma [5.1 to be consistent with our blanket requirement we shall suppose that $T$ is a Hausdorff topological space. We make $\{t\} \times X(t)$ into a Banach space by transfering onto it in the obvious way the structure of $X(t), t \in T$. Put $\mathcal{E} ;=\cup_{t \in T}(\{t\} \times X(t))$ and define $p: \mathcal{E} \rightarrow T$ by $p((t, x)):=t$. For an open subset $V$ of $T$ and an open subset $O$ of $X$ let $\mathcal{U}(V, O):=\{(t, x) \mid t \in V, x \in O \cap X(t)\}$.

Proposition 5.2. The family of all the sets $\mathcal{U}(V, O)$ when $V$ runs through all the open subsets of $T$ and $O$ runs through all the open subsets of $X$ is a base of a topology on $\mathcal{E}$. When $\mathcal{E}$ is endowed with this topology $\xi:=(\mathcal{E}, p, T)$ is a continuous Banach bundle. 
Proof. If $(t, x) \in \mathcal{U}\left(V_{1}, O_{1}\right) \cap \mathcal{U}\left(V_{2}, O_{2}\right)$ then

$$
(t, x) \in \mathcal{U}\left(V_{1} \cap V_{2}, O_{1} \cap O_{2}\right) \subset \mathcal{U}\left(V_{1}, O_{1}\right) \cap \mathcal{U}\left(V_{2}, O_{2}\right) ;
$$

hence the first statement of the proposition. If $V \subset T$ is open then $p^{-1}(V)=$ $\mathcal{U}(V, X)$ so $p$ is continuous. If $V \subset T$ and $O \subset X$ are open then $p(\mathcal{U}(V, O))=$ $V \cap\{t \in T \mid O \cap X(t) \neq \emptyset\}$ so Lemma 5.1 implies that this set is open in $\mathcal{E}$. Thus $\xi$ is a Banach bundle. Let $\left(t_{0}, x_{0}\right) \in \mathcal{E}, r>0$ and set $V:=\left\{t \in T \mid B\left(x_{0}, r\right) \cap X(t) \neq \emptyset\right\}$. The continuity of the norm at $\left(t_{0}, x_{0}\right) \in \mathcal{E}$ follows from

$$
\begin{aligned}
\left(t_{0}, x_{0}\right) \in \mathcal{U}\left(V, B\left(x_{0}, r\right)\right) & =\left\{(t, x) \mid t \in V, x \in B\left(x_{0}, r\right) \cap X(t)\right\} \subset \\
& \left\{(t, x) \in \mathcal{E} \mid\left\|\left(t_{0}, x_{0}\right)\right\|-r<\|(t, x)\|<\left\|\left(t_{0}, x_{0}\right)\right\|+r\right\} .
\end{aligned}
$$

A continuous Banach bundle isomorphic to a Banach bundle as described in Proposition 5.2 is called a uniform Banach bundle. A (continuous) Banach bundle $\xi:=(\mathcal{E}, p, T)$ is called locally uniform if there exists a family of closed subsets $\left\{T_{\alpha}\right\}_{\alpha \in \mathcal{A}}$ of $T$ such that $\left\{\operatorname{Int}\left(T_{\alpha}\right)\right\}_{\alpha \in \mathcal{A}}$ is an open cover of $T$ and each restriction $\xi \mid T_{\alpha}$ is a uniform Banach bundle. One should recall that if $T$ is paracompact or locally compact Hausdorff then each subset $T_{\alpha}$ inherits the same property. If two subspaces of a Banach space have different dimensions then, by [10, the distance between their closed unit balls is at least $1 / 2$. Thus if $\xi:=(\mathcal{E}, p, T)$ is a locally uniform Banach bundle and for some $t \in T$ the fiber $\mathcal{E}(t)$ is $n$-dimensional, $n<\infty$, then there exists a neighbourhood $V$ of $t$ in $T$ such that for each $s \in V$ the fiber $\mathcal{E}(s)$ is $n$-dimensional. It follows that if all the fibers of $\xi$ are finite dimensional and $T$ is locally compact Hausdorff then $\xi$ is locally trivial by [9, Theorem 18.5]. On the other hand there exist uniform Banach bundles with infinite dimensional fibers that are not locally trivial. Indeed, Kadets [14] constructed a Banach space $X$ having subspaces $\left\{X_{n}\right\}_{n=1}^{\infty}$ and $Y$ such that each subspace $X_{n}$ is isometric to the space $\ell^{p_{n}}$ for a sequence $\left\{p_{n}\right\}$ that increases to 2 and $Y$ is isometric to $\left.\ell^{2}\right\}$. Moreover, the the sequence of the closed unit balls of the subspaces $X_{n}$ converges to the closed unit ball of $Y 1$. Thus we have here an example of a uniform Banach bundle whose base space is $\mathbb{N} \cup\{\infty\}$ with the natural topology that is not isomorphic to a locally

\footnotetext{
${ }^{1}$ The author is grateful to Professor E. Gluskin for providing him this reference.
} 
trivial bundle since, as it is well known, the spaces $\ell^{p^{\prime}}$ and $\ell^{p^{\prime \prime}}$ are not isomorphic if $p^{\prime} \neq p^{\prime \prime}$.

We describe now a way to construct locally uniform Banach bundles. Let $T$ be a regular topological space, $\left\{V_{\alpha}\right\}_{\alpha \in \mathcal{A}}$ a cover of $T$ with open non-void subsets, $\left\{X_{\alpha}\right\}_{\alpha}$ a family of Banach spaces such that whenever $V_{\alpha} \cap V_{\beta} \neq \emptyset, X_{\alpha} \cap X_{\beta}$ is a non-trivial closed subspace of $X_{\alpha}$ and of $X_{\beta}$ on which the norms $\|\cdot\|_{\alpha}$ and $\|\cdot\|_{\beta}$ coincide. Denote by $\mathcal{M}_{\alpha}$ the hyperspace of all bounded closed subsets of $X_{\alpha}$ endowed with the Hausdorff metric. Suppose that for each $t \in V_{\alpha}, \alpha \in \mathcal{A}, X(t)$ is a closed subspace of $X_{\alpha}$ and $t \rightarrow X(t)_{1}$ is continuous as a map from $V_{\alpha}$ to $\mathcal{M}_{\alpha}$. Of course, if $t \in V_{\alpha} \cap V_{\beta}$ then $X(t) \subset X_{\alpha} \cap X_{\beta}$. Put

$$
\mathcal{E}_{\alpha}:=\left\{(t, x) \mid t \in V_{\alpha}, x \in X(t)\right\}
$$

and give $\mathcal{E}_{\alpha}$ the topology specified in 5.2 . That is, each pair of open subsets $V \subset V_{\alpha}$ and $O \subset X_{\alpha}$ determines an open subset

$$
\mathcal{U}(V, O):=\left\{(t, x) \in \mathcal{E}_{\alpha} \mid t \in V, x \in O \cap X(t)\right\} .
$$

Observe that if $V_{\alpha} \cap V_{\beta} \neq \emptyset$ then on $\mathcal{E}_{\alpha} \cap \mathcal{E}_{\beta}$ the two relative topologies coincide. Set now $\mathcal{E}:=\cup_{\alpha \in \mathcal{A}} \mathcal{E}_{\alpha}$ and define a topology in $\mathcal{E}$ as follows: $\mathcal{U} \subset \mathcal{E}$ is open if $\mathcal{U} \cap \mathcal{E}_{\alpha}$ is open in $\mathcal{E}_{\alpha}$ for every $\alpha \in \mathcal{A}$. Let $p: \mathcal{E} \rightarrow T$ be the natural map.

Proposition 5.3. With the above notations $\xi:=(\mathcal{E}, p, T)$ is a locally uniform Banach bundle.

Proof. For $t \in V_{\alpha}$ let $A_{t, \alpha}$ be a closed set such that $t \in \operatorname{Int}\left(A_{t, \alpha}\right) \subset A_{t, \alpha} \subset V_{\alpha}$. Clearly the restriction of $\xi$ to $A_{t, \alpha}$ is a uniform Banach bundle.

Continuing with the above notations, if $Y(t)$ is a closed subspace of $X(t), t \in T$, and $t \rightarrow Y(t)_{1}$ is continuous on $T$ then, with $\mathcal{F}:=\{(t, x) \mid t \in T, x \in Y(t)\}$, $\zeta:=(\mathcal{F}, p \mid \mathcal{F}, T)$ is a (locally uniform) Banach subbundle of $\xi$. Indeed, if $V$ is an open subset of $V_{\alpha}$ and $O$ is an open subset of $X_{\alpha}$ then

$$
\begin{aligned}
\{(t, x) \mid t \in V, x \in O \cap X(t)\} \cap\left\{(t, x) \mid t \in V_{\alpha},\right. & x \in Y(t)\}= \\
& \{(t, x) \mid t \in V, x \in O \cap Y(t)\}
\end{aligned}
$$

is a typical open subset of $\mathcal{F}$. Now it is clear that the Banach bundle constructed in Proposition 5.2 is a Banach subbundle of a trivial Banach bundle. Indeed, with 
the notations used there, let $\mathcal{E}^{\prime}:=T \times X$ and $p^{\prime}$ the projection on $T$. Then $\xi$ is by the preceding remarks a Banach subbundle of $\xi^{\prime}:=\left(\mathcal{E}^{\prime}, p^{\prime}, T\right)$. Note that $\mathcal{E}$ is a closed subset of $\mathcal{E}^{\prime}$. To see this, let $\left\{\left(t_{\iota}, x_{\iota}\right)\right\}_{\iota \in \mathcal{I}}$ be a net in $\mathcal{E}$ converging to $(t, x) \in T \times X$. There is no loss of generality if we suppose that $x_{\iota} \in B_{X}\left(0_{X}, 1\right)$, $\iota \in \mathcal{I}$. For each $\iota$ there exists $y_{\iota} \in \mathcal{E}(t)$ such that $\left\|x_{\iota}-y_{\iota}\right\| \leq d\left(\mathcal{E}\left(t_{\iota}\right)_{1}, \mathcal{E}(t)_{1}\right), d$ being the Hausdorff metric. Now $d\left(\mathcal{E}\left(t_{\iota}\right)_{1}, \mathcal{E}(t)_{1}\right) \rightarrow 0$ so $(t, x) \in \mathcal{E}$. On the other hand, not every Banach subbundle of a trivial Banach bundle which has a closed bundle space is a uniform Banach bundle.

Example 5.4. Let $T:=\mathbb{N} \cup\{\infty\}$ with the usual topology, $c_{0}$ the Banach space of all null-convergent sequences of scalars, $\mathcal{E}:=T \times c_{0}$ and $p$ the projection of $\mathcal{E}$ onto $T$. We shall construct a Banach subbundle of the trivial Banach bundle $\xi:=(\mathcal{E}, p, T)$. Denote by $e_{n} \in c_{0}$ the sequence $\left\{\delta_{n}^{k}\right\}_{k=1}^{\infty}$ and let $\mathcal{F}(n)$ be the linear subspace of $\{n\} \times c_{0}$ spanned by $\left(n, e_{1}\right)$ and $\left(n, e_{n}\right)$. We let $\mathcal{F}(\infty)$ be the one-dimensional space spanned by $\left(\infty, e_{1}\right)$. With $\mathcal{F}:=\cup_{t \in T} \mathcal{F}(t)$ the Banach subbundle $\zeta:=(\mathcal{F}, p \mid \mathcal{F}, T)$ of $\xi$ has a closed bundle space. However, the bundle $\zeta$ is not locally uniform because of the drop in dimension at $\infty$.

We have seen in Theorem 3.8 that for a bundle map it is of interest to have a continuous norm in the image bundle. We discuss now conditions that insure that the norm in a quotient bundle is continuous. It may happen that a quotient bundle of a continuous Banach bundle has a discontinuous norm as the following example shows.

Example 5.5. Let $T:=\mathbb{N} \cup\{\infty\}$ with the usual topology, $\xi:=(T \times \mathbb{K}, p, T)$ the one-dimensional trivial bundle over $T$, and $\eta$ its subbundle whose bundle space is $\mathcal{F}:=(\mathbb{N} \times \mathbb{K}) \cup(\{\infty\} \times\{0\})$. Then the quotient bundle space is $(\mathbb{N} \times\{0\}) \cup(\{\infty\} \times \mathbb{K})$ with a non-Hausdorff topology. Hence the quotient bundle is not a continuous Banach bundle.

Question 5.6. Suppose $\eta$ is a quotient bundle of a continuous Banach bundle and the bundle space of $\eta$ is Hausdorff. Must $\eta$ be a continuous Banach bundle?

Proposition 5.7. Let $\xi:=(\mathcal{E}, p, T)$ be a locally uniform Banach bundle and $\xi^{\prime}:=$ $\left(\mathcal{E}^{\prime}, p \mid \mathcal{E}^{\prime}, T\right)$ a locally uniform subbundle. Then the norm on the quotient bundle $\eta:=\left(\mathcal{E} / \mathcal{E}^{\prime}, p^{\prime}, T\right)$ is continuous. 
Proof. A moment's reflection shows that it is enough to consider only the case when $\xi$ and $\xi^{\prime}$ are uniform. Thus we shall suppose that $\xi$ is a bundle as described in Proposition 5.2 keeping the notation used there. We shall also suppose that $Y(t)$ is a closed subspace of $X(t)$ and that the map $t \rightarrow Y(t)_{1}, t \in T$, is continuous into the the space of all closed subsets of the ambient space $X$. Then $\mathcal{E} ;+\cup_{t \in T}(\{t\} \times X(t))$ and $\mathcal{E}^{\prime} ;=\cup_{t \in T}(\{t\} \times Y(t))$. Denote by $q$ the quotient map from $\mathcal{E}$ to $\mathcal{E} / \mathcal{E}^{\prime}$ and let $\alpha \geq 0$; we are going to show that $O:=\left\{y \in \mathcal{E} / \mathcal{E}^{\prime} \mid\|y\|>\alpha\right\}$ is open in $\mathcal{E} / \mathcal{E}^{\prime}$. Suppose then that $y_{0} \in O$ and choose numbers $\beta$ and $\varepsilon$ satisfying $\alpha<\beta<\left\|y_{0}\right\|$ and $0<\varepsilon<\min \left\{1 / 2,1 / 3\left(\left\|y_{0}\right\|-\beta\right)\right\}$. Let $\left(t_{0}, x_{0}\right) \in \mathcal{E}$ with $q\left(\left(t_{0}, x_{0}\right)\right)=y_{0}$ and put $r:=$ $2\left\|x_{0}\right\|+2$. There exists a neighbourhood $V$ of $t_{0}$ in $T$ such that $d\left(Y(t)_{r}, Y\left(t_{0}\right)_{r}\right)<\varepsilon$ if $t \in V$. The set $\mathcal{U}\left(V, B\left(x_{0}, \varepsilon\right)\right):=\left\{(t, x) \mid t \in V, x \in B\left(x_{0}, \varepsilon\right) \cap X(t)\right\}$ is an open neighbourhood of $\left(t_{0}, x_{0}\right)$ and $q\left(\mathcal{U}\left(V, B\left(x_{0}, \varepsilon\right)\right)\right)$ is an open neighbourhood of $y_{0}$, $q$ being an open map. We shall show that $q\left(\mathcal{U}\left(V, B\left(x_{0}, \varepsilon\right)\right)\right) \subset O$ and this will establish our claim about $O$ thereby completing the proof.

Let $(t, x) \in \mathcal{U}\left(V, B\left(x_{0}, \epsilon\right)\right)$ and denote $y:=q(x)$. Our goal is to show that $\|y\|>\alpha$. There exists $\tilde{x} \in Y(t)$ such that $\|y\| \leq\|x-\tilde{x}\|<\|y\|+\varepsilon$. We have $\|\tilde{x}\| \leq r$. Indeed, if we assume by contradiction that $\|\tilde{x}\|>r=2\left\|x_{0}\right\|+2$ then $\|\tilde{x}\|>2\left\|x-\left(x-x_{0}\right)\right\|+2>2(\|x\|-\varepsilon)+2>2\|x\|+1$. But then

$$
\|x-\tilde{x}\| \geq\|\tilde{x}\|-\|x\|>\|x\|+1>\|y\|+\varepsilon,
$$

a contradiction. Now, since $t \in V$ and $\tilde{x} \in Y(t)_{r}$, there exists $\tilde{x}_{0} \in Y\left(t_{0}\right)_{r}$ such that $\left\|\tilde{x}-\tilde{x}_{0}\right\|<\varepsilon$. We have

$$
\begin{aligned}
&\|y\|>\|x-\tilde{x}\|-\varepsilon \geq\left\|\left(\tilde{x}-\tilde{x}_{0}\right)+\left(\tilde{x}_{0}-x_{0}\right)\right\|-\left\|x_{0}-x\right\|-\varepsilon \geq \\
&\left\|\tilde{x}_{0}-x_{0}\right\|-\left\|\tilde{x}-\tilde{x}_{0}\right\|-\left\|x_{0}-x\right\|-\varepsilon \geq\left\|y_{0}\right\|-3 \varepsilon>\beta>\alpha
\end{aligned}
$$

and we are done.

The quotient bundle of a uniform Banach bundle may have a continuous norm even when the subbundle that produces it is not a locally uniform subbundle as the following example shows.

Example 5.8. As before we denote by $c_{0}$ the Banach space of all null converging sequences of scalars and $e_{n}:=\left\{\delta_{n}^{k}\right\}_{k=1}^{\infty}, n \geq 1$. Let $T:=\mathbb{N} \cup\{\infty\}$ with the usual 
topology, $\mathcal{E}:=T \times c_{0}$, and $p$ be the projection onto $T$. We let $X_{n}$ be the subspace of $c_{0}$ spanned by $\left\{e_{l}\right\}_{l=1}^{n}, \mathcal{E}^{\prime}(n):=\{n\} \times X_{n}$, and $\mathcal{E}^{\prime}(\infty):=\mathcal{E}(\infty)$. We affirm that $\xi^{\prime}:=\left(\mathcal{E}^{\prime}, p \mid \mathcal{E}^{\prime}, T\right)$ is a subbundle of the trivial bundle $\xi:=(\mathcal{E}, p, T)$. Indeed, suppose $U \subset \mathcal{E}$ is open and $(\infty, x) \in U \cap \mathcal{E}^{\prime}(\infty)$. There are $r>0$ and $m \in \mathbb{N}$ with the property that if $y \in c_{0},\|y-x\|<r$, and $n \geq m$ then $(n, y) \in U$. Let $k \in \mathbb{N}$ be such that $\bar{x} ;=\left(x_{1}, \ldots, x_{k}, 0 \ldots\right)$ satisfies $\|\bar{x}-x\|<r$. Then if $n>\max \{m, k\}$ one has $(n, \bar{x}) \in U \cap \mathcal{E}^{\prime}(n)$. Thus we found a neighbourhood of $\infty$ included in $p(U)$ and the claim is proved.

Clearly $\xi^{\prime}$ is not a locally uniform subbundle because of the disparity in the dimension of the fiber at $\infty$ with the dimensions of the other fibers in any neighbourhood of $\infty$. It remains to show that the norm in the quotient bundle is continuous. Only the continuity at the points of the fiber at $\infty$ must be proven; but since the fiber at $\infty$ of the quotient bundle is trivial this fact is obvious.

\section{Appendix A. Some topological properties of the Bundle space}

We shall now record several topological properties of the bundle space of a Banach bundle. In the following $\xi:=(\mathcal{E}, p, T)$ will denote a Banach bundle.

The first result treats the complete regularity of the bundle space. A result with the same conclusion but under different hypotheses is [11, Lemma 2.1.6].

Proposition A.1. If $\xi$ is a continuous Banach bundle and $T$ is locally paracompact then $\mathcal{E}$ is completely regular.

Proof. First remark that $\mathcal{E}$ is a Hausdorff space since the norm is continuous on it. Let $A$ be a closed subset of $\mathcal{E}$ and $x_{0} \in \mathcal{E}$ with $x_{0} \notin A$. There are an open neighbourhood $V$ of $t_{0}:=p\left(x_{0}\right)$ in $T$ such that $\bar{V}$ is paracompact and a continuous function $h: T \rightarrow[0,1]$ satisfying $h\left(t_{0}\right)=1$ and $h \mid T \backslash V \equiv 0$. Corollary 2.10 yields a section $\varphi$ of $\xi \mid \bar{V}$ such that $\varphi\left(t_{0}\right)=x_{0}$; there exists $\alpha>0$ such that $\{x \in \mathcal{E} \mid p(x) \in V,\|x-\varphi(p(x))\|<\alpha\} \cap A=\emptyset$. Let $\mu:[0, \infty) \rightarrow[0,1]$ be given by

$$
\mu(s):= \begin{cases}1-s / \alpha, & \text { if } 0 \leq s \leq \alpha, \\ 0, & \text { if } \alpha<s .\end{cases}
$$

The function $f: \mathcal{E} \rightarrow[0,1]$ defined by

$$
f(x):= \begin{cases}\mu(\|x-\varphi(p(x))\|) h(p(x)), & \text { if } p(x) \in V, \\ 0, & \text { if } p(x) \notin V\end{cases}
$$


satisfies $f\left(x_{0}\right)=1$ and $f \mid A \equiv 0$ as needed.

We proceed now to discuss a setting in which $\mathcal{E}$ is a Baire space. We begin with some definitions and results from [19. A family $\mathcal{B}$ of non-void open subsets of a topological space is call ed a pseudo-base of the space if every open non-void subset contains an element of $\mathcal{B}$. A topological space $Z$ is called quasi-regular if each nonvoid open set of $Z$ contains the closure of some non-void open set. A topological space $Z$ is called pseudo-complete if it is quasi-regular and there exists a sequence $\left\{\mathcal{B}_{n}\right\}$ of pseudo-bases of $Z$ with the property that if $U_{n} \in \mathcal{B}_{n}$ and $\overline{U_{n+1}} \subset U_{n}$, $n=1,2, \ldots$ then $\cap_{n} U_{n} \neq \emptyset$. It is easily seen that complete metric spaces and locally compact Hausdorff spaces are pseudo-complete. The cartesian product of any family of pseudo-complete spaces is pseudo-complete, see [19, Theorem 6]. Any pseudo-complete-space is a Baire space, see [19, 5.1].

Proposition A.2. Suppose that the base space $T$ is pseudo-complete and locally paracompact. Then $\mathcal{E}$ is a Baire space.

Proof. Let $\left\{\mathcal{B}_{n}\right\}$ be sequence of pseudo-bases of $T$ as in the definition of the pseudocompleteness. Let $\left\{G_{n}\right\}$ be a sequence of open dense subsets of $\mathcal{E}$ and $G$ an open subset of $\mathcal{E}$. By the assumption of local paracompactness of $T$ and [5, p. 10] there are an open subset $V_{1}$ of $T$, a section $\varphi_{1}$ of $\xi$ over $V_{1}$, and a number $\varepsilon_{1}, 0<\varepsilon_{1}<2^{-1}$, such that

$$
U_{1}:=\left\{x \in \mathcal{E} \mid p(x) \in V_{1},\left\|x-\varphi_{1}(p(x))\right\| \leq \varepsilon_{1}\right\} \subset G \cap G_{1} .
$$

From the assumption of pseudo-completeness of $T$ we infer that there exists $W_{1} \in \mathcal{B}_{1}$ such that $W_{1} \subset \overline{W_{1}} \subset V_{1}$. Now there are an open subset $V_{2} \subset W_{1}$ of $T$, a section $\varphi_{2}$ of $\xi$ over $V_{2} \subset W_{1}$, and a number $\varepsilon_{2}, 0<\varepsilon_{2}<2^{-2}$, such that

$$
U_{2}:=\left\{x \in \mathcal{E} \mid p(x) \in V_{2},\left\|x-\varphi_{2}(p(x))\right\| \leq \varepsilon_{2}\right\} \subset U_{1} \cap G_{2} .
$$

There exists $W_{2} \in \mathcal{B}_{2}$ such that $W_{2} \subset \overline{W_{2}} \subset V_{2}$. An obvious induction process shows that for every natural number $n \geq 2$ there exist an open subset $V_{n} \subset W_{n-1}$ of $T$, a section $\varphi_{n}$ of $\xi$ over $V_{n}$, and a number $\varepsilon_{n}, 0<\varepsilon_{n}<2^{-n}$, such that

$$
U_{n}:=\left\{x \in \mathcal{E} \mid p(x) \in V_{n},\left\|x-\varphi_{n}(p(x))\right\| \leq \varepsilon_{n}\right\} \subset U_{n-1} \cap G_{n}
$$

and there exists $W_{n} \in \mathcal{B}_{n}$ such that $W_{n} \subset \overline{W_{n}} \subset V_{n}$. 
We have $\cap_{n=1}^{\infty} V_{n}=\cap_{n=1}^{\infty} W_{n} \neq \emptyset$. Pick $t_{0} \in \cap_{n} V_{n}$. From $\left\{\varphi_{n}\left(t_{0}\right)-\varphi_{n-1}\left(t_{0}\right) \| \leq\right.$ $\varepsilon_{n-1}<2^{n-1}$ we infer that $\left\{\varphi_{n}\left(t_{0}\right)\right\}$ is a Cauchy sequence in the Banach space $p^{-1}\left(t_{0}\right)$; it converges to a point $x_{0}$ in this space. The inequality $\left\|x_{0}-\varphi_{n}\left(t_{0}\right)\right\| \leq \varepsilon_{n}$, $n=1,2, \ldots$, shows that $x_{0} \in \cap_{n=1}^{\infty} U_{n} \subset G \cap \bigcap_{n=1}^{\infty} G_{n}$ and the proof is complete.

We have seen in Propositions 3.9 and 3.12 that it is of interest to know if a given Banach bundle has a paracompact bundle space. We turn now to examining some cases when $\mathcal{E}$ is paracompact or metrizable. Suppose $\xi$ is a uniform Banach bundle.Then, as remarked above in the paragraph preceding Example 5.4 there exists a Banach space $X$ such that $\mathcal{E}$ is a closed subset of $T \times X$. If $T$ is regular and $\sigma$-compact or paracompact and perfectly normal then $T \times X$ is paracompact by [16, Propositions 4 and 5]. Hence in these cases $\mathcal{E}$ is paracompact by [6, p. 218]. With some more work one can obtain the same conclusion when $\xi$ is a locally uniform Banach bundle. Indeed, let $\left\{T_{\alpha}\right\}$ be a family of closed subsets of $T$ such that $\left\{\operatorname{Int}\left(T_{\alpha}\right)\right\}$ is an open cover of $T$ with non-void subsets and the restriction of $\xi$ to each $T_{\alpha}$ is a uniform Banach bundle. In both cases mentioned above $T$ is paracompact so we may suppose that the family of sets $\left\{T_{\alpha}\right\}$ is locally finite. Each $T_{\alpha}$ inherits the properties of $T$ we consider so each $p^{-1}\left(T_{\alpha}\right)$ is paracompact. But $\left\{p^{-1}\left(T_{\alpha}\right)\right\}$ is a locally finite closed cover of $\mathcal{E}$ so $\mathcal{E}$ is paracompact.

Now if $\xi$ is a uniform Banach bundle with a metrizable base space $T$ then $\mathcal{E}$ is metrizable by being a subset of a metrizable cartesian product. Again this remains true for a locally uniform Banach bundle $\xi$. If $\left\{T_{\alpha}\right\}$ is a locally finite closed cover of $T$ as in the previous paragraph then $\left\{p^{-1}\left(T_{\alpha}\right)\right\}$ is a locally finite closed cover of $\mathcal{E}$ with metrizable subspaces. Then a result of Nagata, see [6, p. 204], implies that $\mathcal{E}$ is metrizable.

Proposition A.3. Let $\xi$ be a continuous Banach bundle with a regular base space $T$ that has a countable base. Moreover, suppose that the Banach space $\Gamma_{b}(\xi)$ is separable. Then $\mathcal{E}$ is regular and has a countable base.

Proof. Remark first that $T$ is a metrizable space therefore, by Proposition A.1, $\mathcal{E}$ is completely regular. Now, say that $\left\{V_{m}\right\}$ is a countable base of $T$ and $\left\{\varphi_{n}\right\}$ is a 
sequence dense in $\Gamma_{b}(\xi)$. For $m, n, k \in \mathbb{N}$ denote

$$
\mathcal{U}(m, n, k):=\left\{x \in \mathcal{E} \mid p(x) \in V_{m},\left\|x-\varphi_{n}(p(x))\right\|<1 / k .\right.
$$

We shall show that $\{\mathcal{U}(m, n, k)\}$ is a base of $\mathcal{E}$. Let $U \subset \mathcal{E}$ be open and $x_{0} \in U$.

The Banach bundle $\xi$ is full so by [5, p. 10] there exist $m \in \mathbb{N}, \varphi \in \Gamma_{b}(\xi)$, and $\delta>0$ such that $p\left(x_{0}\right) \in V_{m}, \varphi\left(p\left(x_{0}\right)=x_{0}\right.$, and

$$
U^{\prime}:=\left\{x \in \mathcal{E} \mid p(x) \in V_{m},\|x-\varphi(p(x))\|<\delta\right\} \subset U .
$$

Choose $k \in \mathbb{N}$ such that $1 / k<\delta / 2$ and $\varphi_{n}$ such that $\left\|\varphi-\varphi_{n}\right\|<1 / k$. It is now routine to check that $x_{0} \in U^{\prime} \subset \mathcal{U}(m, n, k)$.

\section{REFERENCES}

1. E. M. Alfsen and E. G. Effros, Structure in Banach spaces, Part II, Annals of Math. 96 (1972), 129-173.

2. C. W. Baker, A closed graph theorem for Banach bundles, Rocky Mountain J. of Math. 12 (1982), 537-543.

3. R. G. Bartle and L. M. Graves, Mappings between function spaces, Trans. Amer. Math. Soc. 72 (1952), 400-413.

4. E. Behrends, M-Structure and the Banach-Stone Theorem, Springer-Verlag, Berlin, 1979.

5. M. J. Dupré and R. M. Gillette, Banach bundles, Banach modules and automorphisms of $C^{*}$-algebras, Pitman Pub Co., New York, 1983.

6. R. Engelking, Outline of general topology, North-Holland, Amsterdam, 1968.

7. J. M. G. Fell, The structure of algebras of operator fields, Acta Math. 106 (1961), 233-280.

8. J. M. G. Fell and R. S. Doran, Representations of *-Algebras, Locally Compact Groups, and Banach*-Algebraic Bundles, vol. 1, Academic Press, Boston, 1988.

9. G. Gierz, Bundles of Topological Vector Spaces and their Duality, Springer-Verlag, Berlin, 1982.

10. V. I. Gurarii, On openings and inclinations of subspaces of a Banach space (in Russian), Teor. Funktsii, Funktsional. Anal. i Prilozhen. 1 (1965), 194-204.

11. A. E. Gutman, Banach bundles in the theory of lattice-normed spaces (in Russian), Trudy Inst. Mat.,Novosibirsk, v. 29 (1995), 63-211.

12. A. E. Gutman, Banach bundles in the theory of lattice normed spaces I, Siberian Adv. Math. 3 (1993), no. 3, 1-55.

13. A. E. Gutman and A. V. Koptev, On the notion of the dual of a Banach bundle, Siberian Adv. Math. 9 (1999), no. 1, 46-98.

14. M. I. Kadets, Note on the gap between subspaces, Funct. Anal. Appl. 9 (1975), 156-157.

15. A. J. Lazar, Continuous Fields of Postliminal $C^{*}$-Algebras, to appear in Rocky Mountain J. Math.

16. E. Michael, A note of paracompact spaces, Proc. Amer. Math. Soc. 4 (1953), 831-838.

17. E. Michael, Continuous Selections. I, Annals of Mathematics, 63 (1956), 361-382.

18. J. R. Munkres, Topology, second edition, Prentice Hall, 2000.

19. J. C. Oxtoby, Cartesian products of Baire spaces, Fund. Math. 49 (1960/61), 157-166.

School of Mathematical Sciences, Tel Aviv University, Tel Aviv 69978, Israel E-mail address: aldo@post.tau.ac.il 